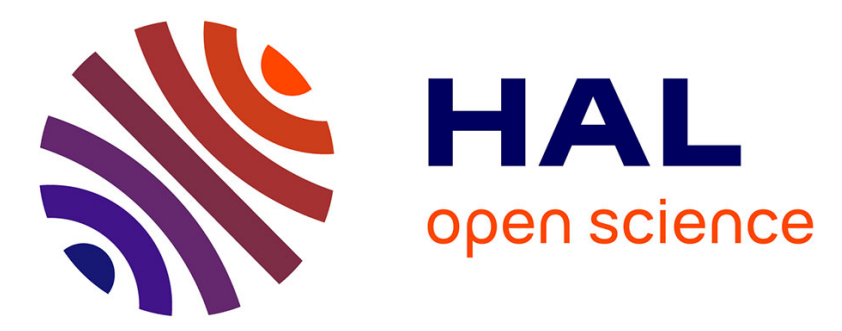

\title{
Vibration-rotation alchemy in acetylene (12C2H2), at low vibrational excitation: From high resolution spectroscopy to fast intramolecular dynamics
}

\author{
David Perry, Anthony Miller, Badr Amyay, André Fayt, Michel Herman
}

\section{- To cite this version:}

David Perry, Anthony Miller, Badr Amyay, André Fayt, Michel Herman. Vibration-rotation alchemy in acetylene $(12 \mathrm{C} 2 \mathrm{H} 2)$, at low vibrational excitation: From high resolution spectroscopy to fast intramolecular dynamics. Molecular Physics, 2010, 108 (07-09), pp.1115-1132. 10.1080/00268971003660874. hal-00596282

\section{HAL Id: hal-00596282 \\ https://hal.science/hal-00596282}

Submitted on 27 May 2011

HAL is a multi-disciplinary open access archive for the deposit and dissemination of scientific research documents, whether they are published or not. The documents may come from teaching and research institutions in France or abroad, or from public or private research centers.
L'archive ouverte pluridisciplinaire HAL, est destinée au dépôt et à la diffusion de documents scientifiques de niveau recherche, publiés ou non, émanant des établissements d'enseignement et de recherche français ou étrangers, des laboratoires publics ou privés. 


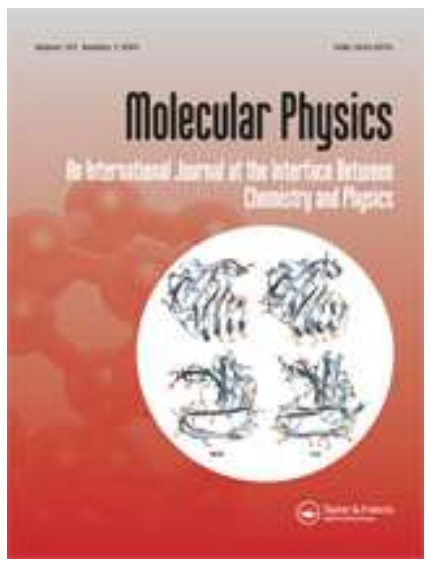

\section{Vibration-rotation alchemy in acetylene (12C2H2), at low vibrational excitation: From high resolution spectroscopy to fast intramolecular dynamics}

\begin{tabular}{|r|l|}
\hline Journal: & Molecular Physics \\
\hline Manuscript ID: & TMPH-2009-0388.R1 \\
\hline Manuscript Type: & Special Issue Paper - In honour of Prof Richard Zare \\
\hline Author: & 25 -Jan-2010 \\
\hline Complete List of Authors: & $\begin{array}{l}\text { Perry, David; University of Akron, Chemistry } \\
\text { Miller, Anthony; University of Akron } \\
\text { AMYAY, Badr; Université libre de Bruxelles, Chimie quantique et } \\
\text { Photophysique } \\
\text { FAYT, André; Université catholique de Louvain } \\
\text { Herman, Michel; Université Libre de Bruxelles, Chimie quantique et } \\
\text { Photophysique }\end{array}$ \\
\hline Keywords: & $\begin{array}{l}\text { IVR in acetylene, Rotational effects in IVR, Overtone spectroscopy } \\
\text { in acetylene, Overtone intensity }\end{array}$ \\
\hline
\end{tabular}

\section{S) ScholaroNE \\ Manuscript Central}




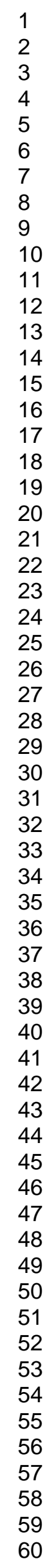

URL: http://mc.manuscriptcentral.com/tandf/tmph 
Vibration-rotation alchemy in acetylene $\left({ }^{12} \mathrm{C}_{2} \mathrm{H}_{2}\right), \tilde{X}^{1} \Sigma_{g}^{+}$at low vibrational excitation:

From high resolution spectroscopy to fast intramolecular dynamics

David Perry, Anthony Miller ${ }^{1}$

B. Amyay ${ }^{2 *}$ A. Fayt ${ }^{3}$, and M. Herman ${ }^{2}$

${ }^{1}$ Department of chemistry

University of Akron

Ohio

USA

${ }^{2}$ Laboratoire de Chimie quantique et Photophysique CP160/09

Faculté des Sciences

Université libre de Bruxelles (U.L.B.)

Ave. Roosevelt, 50

B-1050 Brussels

Belgium

${ }^{3}$ Laboratoire de Spectroscopie Moléculaire Université Catholique de Louvain

Chemin du Cyclotron, 2

B-1348 Louvain-La-Neuve

Belgium

*FRIA researcher

Number of pages: 44

Number of Tables: 0

Number of Figures: 8 (including 23 sub-figures)

Send mail to Prof. M. Herman, mherman@ulb.ac.be

and Prof. D. Perry, dperry@uakron.edu 
1

2

3

4

5

6

7

8

9
The link between energy-resolved spectra and time-resolved dynamics is explored quantitatively for acetylene $\left({ }^{12} \mathrm{C}_{2} \mathrm{H}_{2}\right), \tilde{X}^{1} \Sigma_{g}^{+}$with up to $8,600 \mathrm{~cm}^{-1}$ of vibrational energy. This comparison is based on the extensive and reliable knowledge of the vibration-rotation energy levels and on the model Hamiltonian used to fit them to high precision (B. Amyay, S. Robert, M. Herman, A. Fayt, B. Raghavendra, A. Moudens, J. Thiévin, B. Rowe, and R. Georges, J. Chem. Phys. 131 (2009) 114301114314). Simulated intensity borrowing features in high resolution absorption spectra and predicted survival probabilities in intramolecular vibrational redistribution (IVR) are first investigated for the $v_{4}+v_{5}$ and $v_{3}$ bright states, for $J=2$, 30 and 100. The dependence of the results on the rotational quantum number and on the choice of vibrational bright state reflects the interplay of three kinds of offdiagonal resonances; anharmonic, rotational $l$-type, and Coriolis. The dynamical quantities used to characterize the calculated time-dependent dynamics are the dilution factor $\phi_{\mathbb{d}^{2}}$ the IVR lifetime $\tau_{\mathrm{VR}^{2}}$ and the recurrence time $\tau_{\mathrm{rec}}$. For the two bright states $v_{3}+2 v_{4}$ and $7 v_{42}$ the collisionless dynamics for thermally averaged rotational distributions at $\mathrm{T}=27,270$ and $500 \mathrm{~K}$ were calculated from the available spectroscopic data. For the $7 v_{1}$ bright state, an apparent irreversible decay of is found, In all cases, the model Hamiltonian allows a detailed calculation of the energy flow among all of the coupled zeroth-order vibration-rotation states.
Deleted: the

Deleted: explored
Formatted: Not Highlight

Deleted: quantitatively assess the role

Formatted: Not Highlight Formatted: Not Highlight

Deleted: of three kinds

Formatted: Font: Italic

Formatted: Subscript Formatted: Font: Italic Formatted: Subscript Deleted: IVR from the Deleted: ,

Deleted: states

Deleted: is also predicted from the spectroscopic constants

Deleted: The IVR appears as a

Deleted: in the latter case 


\section{Introduction}

Deleted: $\mathbb{q}$

II

In molecular spectroscopy, when the density of vibrational states is large, a given vibrational bright state that carries oscillator strength from the vibrational ground state may interact with many dark vibrational states. Each dark state then borrows some oscillator strength from the bright state greatly increasing the number of observed features in a high resolution spectrum 1,2 The set of molecular eigenstates that results from the interactions with a single rotational level of the bright state is often referred to as a feature state. Stimulated emission pumping (SEP) and infrared techniques have been applied to obtain eigenstate-resolved spectra of a wide variety of molecules and many insights into the nature of intramolecular vibrational couplings have been obtained 3,4

With the assumption that only one of the zeroth-order vibrational basis states is responsible for all of the observed intensity in a given feature state, the spectra contain enough information to calculate the time dependence following a hypothetical coherent excitation of the whole clump by a short light pulse $\frac{4,5,6, \text { The }}{4 a n}$ initially prepared non-stationary state is the bright state, which subsequently dephases and the vibrational amplitude moves into other zeroth-order basis states. This dynamical process, termed intramolecular vibrational redistribution (IVR), may be characterized by plotting the calculated survival probability of the bright state as a function of time. The time constant for the initial decay of the bright state is $\tau_{I V R}$, and in some cases, the probability of the initial bright state may

Formatted: Superscript
Deleted: ${ }^{1}$
Deleted: ${ }^{2}$
Formatted: Superscript
Deleted: clump

Deleted:

Formatted: Superscript

Deleted: ${ }^{4}$

Formatted: Superscript

Deleted: clump

\begin{tabular}{l} 
Formatted: Superscript \\
Deleted: ${ }^{4}$ \\
\hline Formatted: Superscript \\
Deleted: ${ }^{5}$ \\
Formatted: Superscript \\
Deleted: ${ }^{6}$
\end{tabular}

Formatted: Superscript

Formatted: Superscrip

Deleted:

Deleted: ${ }^{6}$ 
increase again at later times, producing a pattern of recurrences of the bright state with period $\tau_{\text {sec }}$. The time average of the bright state probability, computed out to long times, is the dilution factor $\phi_{d}$.

In general, one finds that low-order anharmonic interactions are strongest 3,7,8. They are noted here $i j / m n$, indicating that quanta of vibrational modes $i$ and $j$ are exchanged for quanta of modes $m$ and $n$. Among them are Fermi interactions (1/22), first demonstrated on $\mathrm{CO}_{2} \stackrel{9}{9}$ which convert one quantum of one vibrational mode (mode 1) into two quanta of another vibrational mode (mode 2). The conversion is two-to-two (11/22) in Darling-Dennison couplings, first reported in

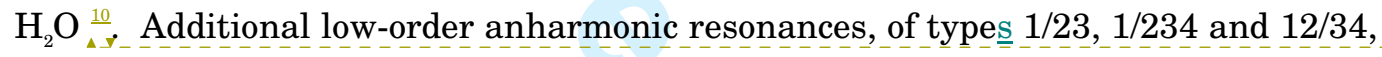
also exist. Higher order interactions may occur indirectly through stepwise low-

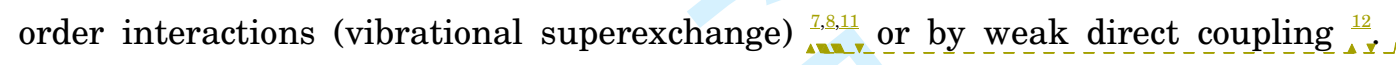
This combination of a few strong interactions with increasing numbers of progressively weaker interactions gives rise to spectra with structure on different frequency scales. Such spectra correspond to bright state decays that occur on a range of timescales $\frac{13,14}{4}$, and have the form of power-law decays $\frac{15,16}{}$

When an observed spectrum has a high density of eigenstates, definitive assignments of the vibrational identity of each eigenstate cannot be achieved. In such cases, one does not have direct information about where the energy goes after it leaves the bright state and statistical treatments of the dynamics are applied $\underline{17,18,19,20,21}$

\begin{tabular}{|c|c|}
\hline Deleted: defir & \\
\hline Formatted & $\ldots[1]$ \\
\hline Deleted: & \\
\hline Formatted & $\ldots[2]$ \\
\hline Deleted: ${ }^{3}$ & \\
\hline Formatted & $\ldots[3]$ \\
\hline Deleted: ${ }^{7}$ & \\
\hline Formatted & $\ldots[4]$ \\
\hline Deleted: ${ }^{8}$ & \\
\hline Formatted & $\ldots[5]$ \\
\hline Deleted: ${ }^{9} \ldots$ I & th $\ldots[6]$ \\
\hline Deleted: ...s & $\ldots[7]$ \\
\hline Formatted & $\ldots[8]$ \\
\hline Deleted: ${ }^{10}$ & \\
\hline Deleted: $^{7}$ & \\
\hline Formatted & $\ldots[9]$ \\
\hline Deleted: ${ }^{8}$ & \\
\hline Formatted & $\ldots[10]$ \\
\hline Deleted: ${ }^{11}$ & \\
\hline Formatted & $\ldots[11]$ \\
\hline Deleted: ${ }^{12}$ & \\
\hline Formatted & $\ldots[12]$ \\
\hline Deleted: weal & \\
\hline Formatted & $\ldots[13]$ \\
\hline Deleted: ${ }^{13}$ & \\
\hline Formatted & $\ldots[14]$ \\
\hline Deleted: ${ }^{14}$ & \\
\hline Formatted & $\ldots[15]$ \\
\hline Deleted: ${ }^{15}$ & \\
\hline Formatted & $\ldots[16]$ \\
\hline Deleted: ${ }^{16}$ & \\
\hline Formatted & $\ldots[17]$ \\
\hline Deleted: ${ }^{17}$ & \\
\hline Formatted & $\ldots[18]$ \\
\hline Deleted: ${ }^{18}$ & \\
\hline Formatted & $\ldots[19]$ \\
\hline Deleted: ${ }^{19}$ & \\
\hline Formatted & $\ldots[20]$ \\
\hline Deleted: ${ }^{20}$ & \\
\hline Formatted & $\ldots[21]$ \\
\hline Deleted: ${ }^{21}$ & \\
\hline
\end{tabular}


The analysis of high resolution vibration-rotation spectra demonstrated the clustering of vibrational states in some molecules, to form polyads or clusters, each assigned to a set of quantum numbers. This structure reliably emerges whenever the ratios of a molecule's vibrational frequencies are approximately the ratios of small integers. Anharmonic resonances connecting the close vibrational states determine the level pattern within each cluster. Each polyad may in fact extend over a broad spectral range. Although there may be significant overlap between polyads, the strongest energy and intensity perturbations strictly arise from interactions within a polyad, at least within excitation ranges as dealt with in the present investigation. Under these circumstances, the polyads are examples of feature states as discussed above. Molecules that exhibit such polyads, when well characterized by conventional spectroscopy, are ideal targets for unraveling the interactions within the feature states and for exploration of the related dynamics.

Vibrational polyads are known to shape the vibrational structure in a number of triatomic species such as $\mathrm{CO}_{2}, \mathrm{H}_{2} \mathrm{O}, \mathrm{N}_{2} \mathrm{O}$ and $\mathrm{OCS}_{4}^{22,23}$. They are also very well characterized in a larger species such as ethyne (acetylene, $\mathrm{C}_{2} \mathrm{H}_{2}$ ) $22,24$. The analysis of conventional and SEP spectra show that there are well-defined polyads at least up to $8,600 \mathrm{~cm}^{-1}$ in ${ }^{12} \mathrm{C}_{2} \mathrm{H}_{2}$ and most probably up to higher energies ${ }_{4}^{22}$ The increasing density of vibrational sub-states with excitation, from less than $1\left(/ \mathrm{cm}^{-1}\right)$ at low energies to dozens around $1 \mu \mathrm{m}$ and 100 's in the visible range ${ }_{4 \rightarrow}^{25}$, generates an interesting set of case studies, with increasing complexity. At intermediate

\begin{tabular}{l} 
Deleted: ${ }^{22}$ \\
Formatted: Superscript \\
Deleted: ${ }^{23}$ \\
Formatted: Superscript \\
Formatted: Superscript \\
Deleted: ${ }^{22}$ \\
\hline Formatted: Superscript \\
Deleted: ${ }^{24}$ \\
Deleted: ${ }^{22}$ \\
Formatted: Superscript \\
Formatted: Superscript \\
Deleted: ${ }^{25}$
\end{tabular}

Deleted: clumps

Deleted: with

Deleted: clumps 


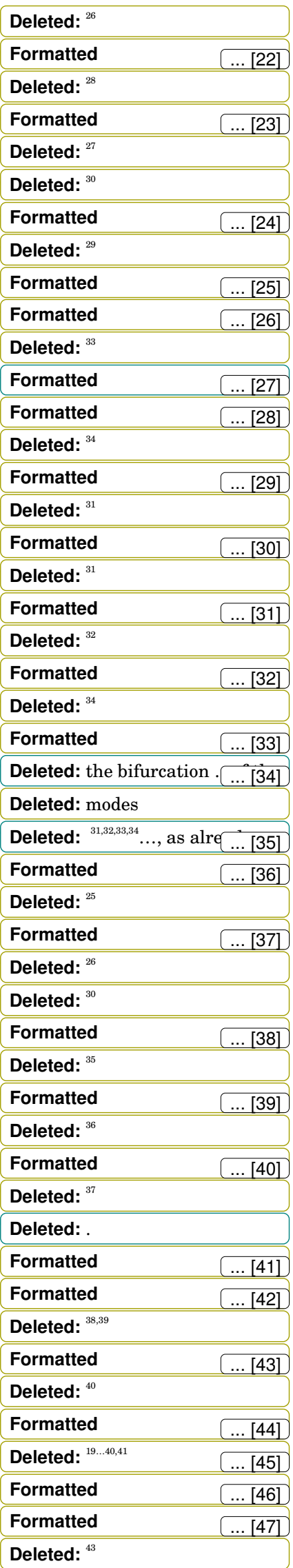

energies, the region up to the threshold for isomerization to vinylidene has yielded a wealth of spectroscopic and dynamical insights. Low-resolution dispersed fluorescence spectra from the $\tilde{A}^{1} A_{u}$ state, reaching low- $J$, low- $k$ states in the 10,000 to $15,000 \mathrm{~cm}^{-1}$ range, have allowed extensive analysis of the various vibrational resonances $\frac{26,27,28,29,30}{4}$ As the total energy increases, these resonances result in new kinds of vibrational motion. ${ }^{31,32,33,34}$ An analysis based on classical mechanics ${ }^{31,34}$ shows that the bending normal modes bifurcate to give rise to new kinds of motions, such as the local bend, the counter rotator, the orthogonal bend, and precessional modes, Vibrational structure in acetylene is thus an ideal test case for exploration of the connection between polyad spectra and dynamics, $25,26,30,35,36,37$.

Following early reports of rotational involvement in IVR $\stackrel{38.39}{4, \text { Lawrance and }}$ Knight $\frac{40}{4 r}$ analyzed the possible roles of Coriolis and centrifugal couplings. These rotational couplings may affect the observed rate of IVR with dependences on the rotational quantum numbers $J$ and $K$ that are characteristic of the particular coupling mechanism $\frac{19,40,41,42}{4,4,4}$, and they may serve to increase the density of coupled levels ${ }^{19,38,42}$. Since Coriolis coupling links the rotational states in steps $\Delta K= \pm 1$, the time required to populate all of the accessible $K$-levels may be very much longer than the decay time $\tau_{\mathrm{IVR}}$ of the bright state ${ }^{20}$.

Recently, a detailed global model has been reported which simultaneously treats both the vibrational and rotational structure in ${ }^{12} \mathrm{C}_{2} \mathrm{H}_{2}$, up to $8,600 \mathrm{~cm}^{-1}{ }^{43}$. 
The reliability and global nature of this spectroscopic Hamiltonian may shed some new light on the vibrational and also rotational aspects of IVR.

The aim in this work is to explore quantitatively the relationship between the

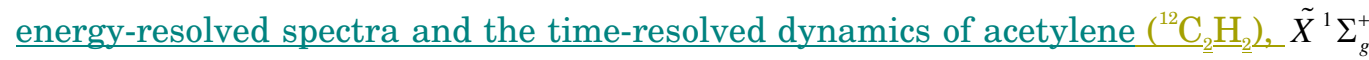
by taking advantage of the detailed knowledge of the vibration-rotation energy levels in this species up to $8,600 \mathrm{~cm}^{-1}$. Specifically, we will examine how the collisionless time-dependent dynamics are affected by (i) the choice of the bright vibrational state, (ii) the extent of rotational excitation, and (iii) the excitation of a thermal ensemble of rotational levels.

Section II of this paper reviews the means by which time-dependent dynamics are deduced from frequency-resolved spectroscopic data. After recalling significant features of the global model in section III, the role of basic intramolecular couplings is illustrated in a series of pedagogical case studies in section IV, both in energy and time domains. Then in section $\mathrm{V}$, the dynamical behavior of thermal distributions of $J$-levels is examined for two case studies, before concluding in section VI.

\section{Spectroscopy and Dynamics}

\begin{tabular}{l} 
Deleted: to link quantitatively \\
the energy- and time-resolved \\
approaches, including the \\
rotational dependence, \\
Deleted: extensive and precise \\
Deleted: acetylene \\
Deleted: $\left({ }^{12} \mathrm{C}_{2} \mathrm{H}_{2}\right), \tilde{X}^{1} \Sigma_{g}^{+}$ \\
\hline Deleted: \\
Deleted: . 1 \\
\hline Formatted: Superscript \\
Deleted: in order to explore the \\
quantitative link
\end{tabular}

Deleted: to link quantitatively Deleted: Furthermore, it allows for the first time a quantitative exploration of the role of rotational degrees of freedom in acetylene IVR. 
The usual unperturbed / perturbed or zeroth-order / eigenstate scheme for a 2 by 2 perturbation is recalled in Figure 1 , with notations similar to Xu et al 44 We use the convention that states with lower numbering (I/1) have lower energy than states with higher numbering (II/2). Such a scheme is used in high resolution, energy-resolved spectroscopy to explain intensity borrowing that results from anharmonic or Coriolis mixing of two zeroth-order rotation-vibration states. In this case $_{2}$ the mixing is often presented as

$$
\begin{aligned}
& \psi^{I}=a \psi_{1}^{0}-b \psi_{2}^{0} \\
& \psi^{I I}=b \psi_{1}^{0}+a \psi_{2}^{0}
\end{aligned}
$$

where the coefficients $a$ and $b$ are identical to those in Figure 1. Assuming the lower energy zeroth-order state, $\psi_{1}^{0}$ is the bright state $(1 \equiv \mathrm{B})$ and the higher energy one, $\psi_{2}^{0}$ a dark state $(2 \equiv \mathrm{D})$, the experimental transitions from the vibrational ground state GS obey the selection rules

$$
\begin{aligned}
& \left|\left\langle\psi_{B}^{0}|\mu| \psi_{G S}\right\rangle\right|^{2} \neq 0 \\
& \left|\left\langle\psi_{D}^{0}|\mu| \psi_{G S}\right\rangle\right|^{2}=0
\end{aligned}
$$

and the relative intensities of the $\mathrm{I} \leftarrow$ GS and II $\leftarrow$ GS IR bands are, respectively

$$
\begin{aligned}
& I^{I} \propto a^{2} \\
& I^{I I} \propto b^{2}
\end{aligned}
$$

\begin{tabular}{|l|}
\hline Deleted: from \\
Deleted: $^{44}$ \\
Formatted: Superscript
\end{tabular}

Deleted: issues
Deleted:

Formatted: Tabs: Not at $36 \mathrm{pt}+$ $72 \mathrm{pt}$ 
with $a^{2}+b^{2}=1$. Thus the intensities $I^{I}$ and $I^{I I}$ tell how much of the zeroth-order bright state is contained in each of the eigenstate wavefunctions, as represented in Figure 1 by the red fractions in $\psi^{I}$ and $\psi^{I I}$.

This scheme is also used in time-resolved spectroscopy when coherently exciting the two eigenstates $\psi^{I}$ and $\psi^{I I}$ with a short laser pulse and following the evolution with time of the total, non stationary wavefunction $4.6,45,46,47,48$,

$$
\Psi(t)=a \psi^{I} \exp \left(-i E^{I} t / \hbar\right)+b \psi^{I I} \exp \left(-i E^{I I} t / \hbar\right)
$$

The time-dependent probability of finding the system in the bright and dark zerothorder states is oscillatory

$$
\begin{aligned}
& P_{B}(t)=\left|\left\langle\psi_{B} \mid \Psi(t)\right\rangle\right|^{2}=1-2 a^{2} b^{2}(1-\cos (\delta \varepsilon t / \hbar)) \\
& P_{D}(t)=\left|\left\langle\psi_{D} \mid \Psi(t)\right\rangle\right|^{2}=2 a^{2} b^{2}(1-\cos (\delta \varepsilon t / \hbar))
\end{aligned}
$$

where $\delta \varepsilon=E^{I I}-E^{I}$, and the oscillation period is $\tau_{\underline{\underline{v e c}}}=h / \delta \varepsilon$.

This approach can be extended to a more complicated set of interactions involving $n$ basis states ${ }^{5,6}$. In this case, the basis functions $\psi_{j}^{0}$ can be expressed as a linear combination of the $n$ eigenstates $\psi^{k}$ :

$$
\psi_{j}^{0}=\sum_{k=1}^{n} c_{j k} \psi^{k}
$$

If we assume that only one of the basis states, say $j=1$, carries oscillator strength Deleted: s from the ground state, then the relative intensities of the eigenstates are 


$$
I_{k} \propto c_{1 k}^{2}
$$

and the time-dependent wavefunction following a coherent excitation is

$$
\Psi(t)=\sum_{k=1}^{n} c_{1 k} \psi^{k} \exp \left(-i E^{h} t / \hbar\right)
$$

At $t=0$, the bright state is prepared, $\Psi(0)=\psi_{1}^{0}$, but thereafter the time-dependent probability of the various basis states may be calculated as

$$
P_{j}(t)=\left|\left\langle\psi_{j}^{0} \mid \Psi(t)\right\rangle\right|^{2}
$$

When $j=1$, this yields $P(t)$, the survival probability of the bright state. This timedependence is in general more complicated than the simple oscillatory pattern given Formatted: Font: Italic Formatted: Font: Italic Formatted: Subscript Formatted: Font: Italic in Eq. (5) and involves movement of vibrational amplitude among the various coupled rotation-vibration states, that is, IVR.

There are a number of physical phenomena that are not included in the present treatment. In particular, we include no effects, such as collisions or infrared $\underline{\text { radiative lifetimes, that would impart a finite lifetime and a homogeneous line }}$ width to individual eigenstates. Hyperfine structure is not considered here. Below, we do include a thermal distribution of rotational levels as an approximate treatment of the inhomogeneous rotational structure, but do not consider Doppler broadening. The neglected effects would cause $P_{1}(t)$ to decay to zero or at least damp out its fluctuations. As calculated from the above equations, $P_{d}(t)$ will continue indefinitely to fluctuate around a finite average value.

Deleted: a

\begin{tabular}{|l|}
\hline Deleted: , but as \\
\hline Formatted: Font: Italic \\
\hline Formatted: Subscript \\
\hline Formatted: Font: Italic \\
\hline
\end{tabular}

49

50

51

52

53

54

55

56

57

58

59

60 
Three different measures are used in this paper to characterize the timedependent dynamics. The first is the dilution factor ${ }^{49,50,51} \phi_{0,0}$ which is determined by the way the bright state character is distributed among each of the eigenstates,

$$
\begin{aligned}
\phi_{d} & =\sum_{k=1}^{n} c_{1 k}^{4} \\
& =\sum_{k=1}^{n} I_{k}^{2} /\left(\sum_{k=1}^{n} I_{k}\right)^{2}
\end{aligned}
$$

and is consequently related to the spectral intensities through Eq. (7). The effective number of coupled states is $1 / \phi_{d}$. For an unperturbed state, $\phi_{d}=1$, and if all $n$ eigenstates appeared in the spectrum with equal intensity, we would have $\phi_{d}=1 / n$. The dilution factor can be obtained from the time-dependent dynamics as the timeaveraged probability of the bright state, $P(t)$, where the average extends out to long times, $_{2}$

$$
\phi_{d}=\lim _{t \rightarrow \infty} \frac{1}{t} \int_{0}^{t} P_{1}(t) d t
$$

The second measure, IVR lifetime ${ }_{-1}^{3} \tau_{\text {IVR2 }}$ is usually defined as the time required for the bright state probability $P_{1}(t)$ to decay from its initial value, $P_{1}(0)=1$, to $1 / e$. Since in general the form of $P_{1}(t)$ is non-exponential, the $1 / e$ value is simply a convenient reference point to take as a measure of the rate of IVR. In the present work, where we are dealing with the very sparse limit, we define $\tau_{\text {IVR }}$ as the time for 
1

2

3

4

5

6

7

8

9

10

11

12

13

14

15

16

17

18

19

20

21

22

23

24

25

26

27

28

29

30

31

32

33

34

35

36

37

38

39

40

41

42

43

44

45

46

47

48

49

50

51

52

53

54

55

56

57

58

59

60
$P_{1}(t)$ to decay to $\phi_{d}+(1 / e)\left(1-\phi_{d}\right) \cdot \frac{51}{2}$ In the limit of many coupled levels $\left(\phi_{d} \rightarrow 0\right)$, this reduces to the usual definition of $\tau_{I V R^{*}}$

The final measure is the recurrence time $\tau_{r e q}$, which is just the oscillation period (Eq. (5)) when there are only two interacting states. When there are more interacting levels, multiple recurrence times may be evident in a single $P_{1}(t)$ curve ${ }_{*}$

The spectrum of eigenstates resulting from the excitation of a single ground state rotational level is referred to as a "feature state" or "clump" and represents a kind of homogeneous broadening. Thus, the ensuing dynamics are also called "homogeneous". This will be contrasted below with an inhomogeneously broadened spectrum resulting from of the excitation of a thermal distribution of rotational $\underline{\text { levels and the ensuing inhomogeneous dynamics. }}$ .

\section{A global spectroscopic Hamiltonian}

The conventional normal mode numbering for acetylene is used throughout this paper. Modes 1 to 5 correspond to the symmetric $\mathrm{CH} \sigma_{g}^{+}\left(v_{1}\right)$ and $\mathrm{CC} \sigma_{g}^{+}\left(v_{2}\right)$ stretches, the antisymmetric $\mathrm{CH}$ stretch $\sigma_{u}^{+}\left(v_{3}\right)$, and the trans $-\pi_{g}\left(v_{4}\right)$ and cis $-\pi_{u}$ $\left(v_{5}\right)$ doubly degenerate bends, respectively. The bends are characterized by the usual vibrational angular momentum quantum numbers, $l_{4}$ and $l_{5}$, with $k=l_{4}+l_{5}$. In this paper, state refers to a vibrational state characterized by the $\left(v_{1} v_{2} v_{3} v_{4} v_{5}\right)$ set

\footnotetext{
In this paper, state refers to a vibrational state characterized by the $\left(v_{1} v_{2} v_{3} v_{4} v_{5}\right)$ set
}

Deleted:

Deleted: ${ }^{51}$

Formatted: Superscript

Formatted: Indent: First line: $10.35 \mathrm{pt}$

Formatted: Font: Not Italic, Not Superscript/ Subscript

Deleted: II

Deleted:

Deleted: II 
of quantum numbers. Sub-state indicates a $l_{i}$-component of a state notated as either $v_{1} v_{2} v_{3} v_{4} v_{5}, l_{4} l_{5}$ or $v_{1} v_{2} v_{3} v_{4}^{l_{4}} v_{5}^{l_{5}}$. The symmetry labels include $e / f \frac{52}{5-v_{-}}$and $u / g$. Level refers to a specific $J$-value of a state or sub-state.

Over the last years, a global vibration-rotation model of acetylene $\left({ }^{12} \mathrm{C}_{2} \mathrm{H}_{2}\right)$ in the ground electronic state has emerged from a series of investigations taking into account the complete set of experimental data published in the literature, now up to $8,600 \mathrm{~cm}^{-1} \frac{43}{4}$ This model is based on the block diagonalization of the vibrationrotation Hamiltonian into polyads $\frac{24,53}{2-n}$

Two vibrational quantum numbers can be defined, $N_{r_{-}}=5 v_{1}+3 v_{2}+5 v_{3}+v_{-}+v_{5}$ and $N_{s}=v_{1}+v_{2}+v_{3} \frac{54,55,56}{4}$. Symmetrization factors the Hamiltonian into $e$ and $f$ blocks $\mathrm{s}^{57}$ Rotational $l$-type resonance terms connect sub-states with either even $(e)$ or odd $(o)$ values of $k$. Polyads thus include sub-states with either even or odd $k$ values. That is $\Sigma(k=0), \Delta(k=2), \Gamma(k=4)$, etc. are in a separate polyad from $\Pi(k=1), \Phi(k=3)$. etc. Vibrational $l$-type resonance terms are also included. The resulting vibrationrotation matrices are diagonal in $J$. A complete polyad label is $\left\{N_{s}, N_{r}, k_{e / o}, e / f, u / g, J\right\}$.

The sub-states within a polyad are connected by a restricted set of anharmonic resonances, namely 11/33, 14/35, 15/34, 1/244, 1/255, 3/245 and 44/55, all determined from the spectral analysis $\frac{24,53}{4}$ The reader is referred to Table I in Fayt et al $\frac{58}{\Delta \frac{5}{2}}$ for the definition of the matrix elements and parameters in the model.
Deleted: ${ }^{52}$

Formatted: Superscript

\begin{tabular}{|c|}
\hline Formatted: Superscript \\
\hline Deleted: ${ }^{43}$ \\
\hline Deleted: ${ }^{53}$ \\
\hline Formatted: Superscript \\
\hline Deleted: ${ }^{24}$ \\
\hline Formatted: Superscript \\
\hline Deleted: pseudo \\
\hline Formatted: Superscript \\
\hline Deleted: ${ }^{54}$ \\
\hline Formatted: Superscript \\
\hline Deleted: ${ }^{55}$ \\
\hline Formatted: Superscript \\
\hline Deleted: ${ }^{56}$ \\
\hline $\begin{array}{l}\text { Deleted: The } e \text { and } f \text { partition } \\
\text { is applied to build the } \\
\text { Hamiltonian in the } \\
\text { symmetrised basis }\end{array}$ \\
\hline Formatted: Superscript \\
\hline Deleted: ${ }^{57}$ \\
\hline Formatted: Superscript \\
\hline Deleted: ${ }^{24}$ \\
\hline Formatted: Superscript \\
\hline Deleted: ${ }^{53}$ \\
\hline $\begin{array}{l}\text { Deleted: As in the introductory } \\
\text { section, the } i j / m n \text { labelling } \\
\text { refers to an interaction coupling } \\
\text { vibrational states with }\end{array}$ \\
\hline$\Delta v_{i}=\Delta v_{j}= \pm 1$ and \\
\hline$\Delta v_{m}=\Delta v_{n}=\mp 1$ \\
\hline Formatted: Superscript \\
\hline Deleted: ${ }^{58}$ \\
\hline
\end{tabular}


The Coriolis interaction, of the 2/444 type in particular, was recently introduced in the model by Fayt and coworkers ${ }_{43}^{43}$ The resulting couplings are weak but significant. Since they introduce matrix elements with $\Delta k= \pm 1$, the even and odd $k$-blocks are now coupled. The $N_{s}$ quantum number is no longer well-defined and the resulting polyads are larger. They are labelled $\left\{N_{r}, e / f, u / g\right\}$.

This global model was applied to all 15,562 published vibration-rotation lines in ${ }^{12} \mathrm{C}_{2} \mathrm{H}_{2}$ up to $8,600 \mathrm{~cm}^{-1}$. The data base includes rotational levels up to $J=68$ for some sub-states as well as data from highly excited trans-bend states, from Field and coworkers $\frac{59}{4}$. All lines could be reproduced within their stated measurement accuracy $(3 \sigma)$, meaning typically better than $0.001 \mathrm{~cm}^{-1}$, except for a limited number of them (8\%), These problems are all local and attributed either to measurement problems, due to line overlap or lower line intensity, or to Coriolis perturbation, that cannot be fixed without a dedicated reanalysis of the literature spectra. We are confident that such small errors do not affect the trends emerging from this work. In all, 331 vibration-rotation parameters were determined from the fit.

The present investigation is meant to highlight the basic processes. Therefore, the case studies to be presented here were selected to be well within the energy range $\left(\leq 8,600 \mathrm{~cm}^{-1}\right)$ of the data fit by the global model.

\begin{tabular}{|l|}
\hline Formatted: Superscript \\
\hline Deleted: ${ }^{59}$ \\
\hline Deleted: Except for a limited \\
number (8\%) of local ized errors \\
in the fitproblems, \\
\hline Deleted: a \\
\hline Deleted: but \\
\hline Deleted: . \\
\hline Deleted: However, w \\
\hline
\end{tabular}

Deleted: do not believe that

Deleted: considered in

Deleted: The results nevertheless involve energy predictions from the model which may fail to exactly match local, $J$-specific features such as avoided crossings, at the spectroscopic accuracy. Such problems are however not believed to affect the trends emerging from the present

investigation. 


\section{Basic intramolecular couplings}

The definition of the zeroth-order states is a critical issue. We include in this concept the states whose energy results from all of the diagonal matrix elements in the global model, in the symmetrised e/f basis set as defined by Fayt and coworkers 57. Therefore, the implicit definition used here is that the zeroth order states are those states that have the diagonal energies of the global model as their eigenvalues. In a multidimensional problem, even as small as for acetylene, it is a challenge to write out an explicit form for such "implicit" basis functions. Indeed this challenge constitutes one of the conceptual limitations of effective Hamiltonians derived from fitting spectroscopic data.

The implicit set of zeroth-order basis states incorporates all of the major terms defining the vibration-rotation energies in linear molecules, including the role of diagonal vibrational ${ }_{4,61}^{61}$ and rotational $l$-type resonances, shaping the regularly evolving vibrational and rotational energy spacings with increasing $v_{i}$ and $J$ excitation. This set allows convenient implementation of the major transition selection rules, including identification of bright state. The remaining terms in the Hamiltonian are off-diagonal and arise from anharmonic interaction, Coriolis coupling and rotational $l$-type resonance. Their introduction into the Hamiltonian matrix provides coupling between the basis states and hence defines eigenstates. The role of each of these terms in coupling the basis states and in the timedependent dynamics is investigated in the present section. Only the role of the
Deleted: Here, we start with

\begin{tabular}{l} 
Deleted: TIn the present global \\
model, the diagonalse elements, \\
thus including the role of \\
diagonal vibrational ${ }^{60,61}$ and \\
rotational $l$-type resonances \\
Deleted: \\
Deleted: shape the regularly \\
evolving vibrational and \\
rotational energy spacings with \\
increasing $v_{i}$ and $J$ excitation. \\
\hline Deleted: Such a basis \\
\hline Deleted: \\
Formatted: Superscript \\
\hline Formatted: Superscript \\
\hline Deleted: and thusand it \\
\hline
\end{tabular}

Formatted: Superscript

Deleted: ${ }^{57}$, and then . include all of the diagonal matrix elements in the global model

Deleted: and thusand it

Deleted: coupling 
dominant term in each coupling case is discussed in the text, but all terms are included in the calculations.

As pointed out before, zeroth-order sub-states are labeled $\left(v_{1} v_{2} v_{3} v_{4} v_{5}, l_{4} l_{5}\right)$. Eigenstates are labeled using Roman numerals, numbered from lowest energy to highest.

$$
\text { |. }
$$

\section{A. Rotational $l$-type resonance}

The rotational $l$-type resonance connects sub-states related by $\Delta k= \pm 2$, with matrix elements scaling as $J(J+1) \frac{62,63,64,65}{4}$ The resultant mixing of the basis states is illustrated in Figure 2 for the $\left\{0,2, k_{e}, e, u\right\}$ polyad. This polyad contains two zerothorder sub-states, $(00011,1-1), \Sigma_{e u}$ and $(00011,11), \Delta_{e u}$. The first of these is the only one allowed by the conventional $\Delta k=0, \pm 1$ IR selection rules from the ground state (GS), thus leading to the (00011,1-1), $\Sigma_{e u} \leftarrow$ GS transition. It is therefore the bright sub-state for IR absorption from the GS.

The rotational $l$-type resonance introduces $|\Delta k|=2$ interaction terms connecting the zeroth-order states,

$$
\left\langle 00011, \Sigma_{e}\left|H_{e f f}\right| 00011, \Delta_{e}\right\rangle=\frac{1}{2}\left(q_{4}+q_{5}\right)[J(J+1)[J(J+1)-2]]^{1 / 2} .
$$

We consider only the $e$-levels defining the two eigenstates I and II. Their energies were calculated from the global model. The reduced energies presented in Figure
Deleted: detailed Deleted: while

Deleted: For completeness, one should add that off-diagonal vibrational $l$-type resonance terms also contribute to eigenstates. They are included in the Hamiltonian, but their role is not considered further in the discussion below.

Deleted: II

Formatted: Indent: First line: 0 pt
Deleted: 11

Deleted: here
Deleted: , only,
Deleted: sub-


2a were obtained by subtraction of the rotational energy contribution determined using a selected set of B and D parameters, identical for both sub-states.

The separation between the two sets of eigenenergies is determined by the parameters $\left(\right.$ in $\mathrm{cm}^{-1}$ ) $\mathrm{q}_{4}=-5.23110^{-3}$ and $\mathrm{q}_{5}=-4.69810^{-3} \frac{43}{4 \mathrm{r}}$ and is $J(J+1)$-dependent in agreement with Eq._(12). The zeroth-order sub-states are also presented on Deleted: Formatted: Superscript Deleted: 11 Figure 2a, and identified on the left using the $\left(v_{1} v_{2} v_{3} v_{4} v_{5}, l_{4} l_{5} e u\right)$ label. The $J$ dependence of the rotational $l$-type resonance is obvious. The bright sub-state corresponds to the solid line, in red, The same presentation will occur in all similar graphs.

The amount of the bright zeroth-order sub-state in eigenstates I and II was calculated from the eigenvectors in the matrix diagonalisation process. It is quantified in Figure 2b. The zeroth-order bright character is dominant in I (100\%) at $J=0$ and regularly decreases to about $53 \%$ at $J=100$, with II following a similar but opposite trend.

The mixing between the two zeroth-order wavefunctions by the rotational $l$ type resonance perturbation results in more consequences, which are illustrated in the intensity and time domains:

i) Spectral intensities: Figure 2c presents the relative intensity of the $R$ branches of the I $\leftarrow$ GS and II $\leftarrow$ GS IR absorption bands. For clarity, the 3:1 intensity alternation due to ortho and para acetylene $\frac{24,53}{4}$ has been cancelled by 
multiplying the lower intensity lines by a factor 3 . They were simulated for temperature conditions high enough $(500 \mathrm{~K})$ for high $J$-levels to be populated in the GS. As a consequence of the rotational $l$-type resonance, intensity borrowing occurs ${ }_{2}$ transferring some of the intensity of the $\mathrm{I} \leftarrow$ GS band to the II $\leftarrow$ GS band. The intensity transfer is greater at high $J$-values, as expected from the increasing strength of the rotational $l$-type resonance matrix elements (Eq. (12)). The overall intensity transfer remains modest.

ii) Survival probability: Figure $2 \mathrm{~d}$ presents the survival probabilities in the zeroth-order bright sub-state calculated for three selected values of $J(2,30$ and 100). The same color and marker style convention is used in Figures $2 \mathrm{a}$ and $2 \mathrm{~d}$ for the zeroth-order sub-states. In this 2-state case, the time-dependences follow Eq. (5). At $J=2$, the wave packet remains in the bright zeroth-order sub-state. As $J$ increases, oscillation occurs, with the other zeroth-order sub-state participating. The frequency and amplitude of the oscillation increase with $J$, again as expected from the rotational $l$-type resonance matrix elements (Eq. (12)). The period of the oscillation, $\tau_{\underline{r e c}}=h / \delta \varepsilon_{\text {, }}$ defined in Eq. (5) thus decreases as the energy difference $(\delta \varepsilon)$ increases. The dilution factor $\left(\phi_{d}\right)$ evolves from the limit of weak interaction $\left(\phi_{d}=1\right)$ at $J=2$ to approach the limit of strong interaction between the two basis states $\left(\phi_{d}\right.$ $=0.5)$ at $J=100$.
Deleted: 11 


\section{B. Anharmonic resonances}

The next case study is the $\left\{1,5, k_{e}, e, u\right\}$ polyad, which contains three zerothorder sub-states, $(00100,00), \Sigma_{e u},(01011,1-1), \Sigma_{e u}$ and $(01011,11), \Delta_{e u} \cdot$ The same rotational $l$-type resonance perturbation as just discussed is again active. In addition to rotational $l$-type resonance, the anharmonic $3 / 245$ coupling $\frac{66}{4}$ connects Deleted: ${ }^{66}$,

Formatted: Superscript

Deleted: 12

with $\left|\mathrm{K}_{3 / 245}\right|=18.3 \mathrm{~cm}^{-1} \stackrel{43}{4}$

This additional coupling is strong, with $J$-independent matrix elements; however, the mixing is slightly $J$-dependent. In a second-order perturbation scheme, the mixing coefficients are related to the energy difference between the two interacting levels (Fig. 1), which varies for each pair of $J$-levels according to the vibrational dependence of the rotational constants. A reduced energy graph is presented in Figure 3a. The zeroth-order sub-states are also presented and identified on Figure 3a. At very low $J$-values, $(000100,00) \Sigma_{e u}$ is slightly higher in energy than $(01011,1-1), \Sigma_{\text {eu }}$.

In order to allow for a direct comparison with the above case study, we consider first the fate of the $(01011,1-1), \Sigma_{e u}$ zeroth-order sub-state, which is taken 
for the moment as the bright state, and therefore plotted as a solid red line in Figure 3a. With allowance for the increased vibrational energy, the behavior would be very similar to the one exemplified in Figure 2 for the $(00011,1-1), \Sigma_{\text {eu }}$ zerothorder sub-state since they both involve the $v_{4}+v_{5}$ vibrational combination. The interplay is however now more complex thanks to the anharmonic resonance bringing in the third sub-state. Compared to situation in Figure 2a, the anharmonic coupling now leads to a significant repulsion between the two zerothorder sub-states close to $3290 \mathrm{~cm}^{-1}$, while the rotational $l$-type resonance acts more strongly at higher $J$-values. The situation is further detailed in Figure $3 b$, which quantifies the amount of the zeroth-order sub-state in eigenstates I to III. The fraction of the zeroth-order bright state is nearly identical in I and II for the lowest $J$-values, then increases slightly for II up to $J=45$, where it falls below I again. As $J$ increases further, the zeroth-order bright wavefunction is shared mainly between I and III. This behavior looks thus different from the previous case study because at low $J$-values the anharmonic resonance dominates. At high $J$-values, it is more similar to the previous case because the $J$-dependent rotational $l$-type resonance becomes the dominant interaction.

In this 3-level system, the time-dependence determined by Eqs. (8) and (9) is more complicated than that given in Eq. (5). The time-dependent probabilities in Figure $3 c$ reflect the fractionation discussed above. The probability first oscillates between the anharmonically coupled, $(01011,1-1)$ and $(00100,00) \Sigma_{\text {eu }}$ zeroth-order 
sub-states $\left(J=2\right.$, top graph), while the third $(01011,11) \Delta_{e u}$ zeroth-order sub-state is more and more involved $(J=30$, middle graph) and even dominates over $(00100,00)$ at high $J$-values $(J=100$, bottom graph). Thus the rotational $l$-type resonance takes over as $J$ increases.

The set of graphs is repeated in Figure 4, but now selecting the bright substate for IR absorption from the GS, i.e. $(00100,00), \Sigma_{e u}$. This sub-state involves only one vibrational quantum excitation, as compared to three for $(01011,1-1), \Sigma_{e u}$. Furthermore, it corresponds to a $\mathrm{CH}$ stretch, which is expected to result in a strong absorption band. Figure $4 \mathrm{a}$ is thus identical to Figure $3 \mathrm{a}$, but for the selection of the zeroth-order bright sub-state. The related line color and marker style were adapted accordingly. Figure $4 \mathrm{~b}$ presents the fraction of this new zeroth-order bright sub-state in the eigenstates. Figure 4c shows the resulting intensity borrowing, using the same convention for the ortho:para relative line intensities, as in Figure 2c. It occurs as in the previous case study (Fig. 2c), but is now distributed over three bands in agreement with the mixing scheme. In other words the zeroth-order bright character is equally shared by the I $\leftarrow$ GS and II $\leftarrow$ GS bands at low $J$, then I $\leftarrow$ GS gains intensity up to $J=55$, after which II $\leftarrow$ GS dominates. The band III $\leftarrow$ GS is weaker but receives about $2 \%$ of bright character for $35<J<45$. The population factor, in Figure 4c, makes this band appear stronger between $J=20$ and 30 , at $500 \mathrm{~K}$. 
The survival probability for the infrared-active bright state is presented in Figure $4 \mathrm{~d}$ for three selected $J$-values (2, 30 and 100). As could be expected from the previous graphs in Figure 4, the wavepacket first oscillates between the bright substate and the anharmonically coupled state, $(01011,1-1), \Sigma_{e u}$ (top graph). However, now the third zeroth-order state, $(01011,11), \Delta_{e u}$, is only indirectly coupled to $(00100,00), \Sigma_{\text {eu }}$ through the $(00100,00) \stackrel{3 / 245}{\longleftrightarrow}(01011,1-1) \stackrel{\text { rot.l-res. }}{\longleftrightarrow}(01011,11)$ interaction scheme. It therefore comes in only slightly, for intermediate $J$-values (middle graph). At high $J$-values (bottom graph), the reduced amplitude of the oscillation shows that the zeroth-order $(00100,00) \Sigma_{e u}$ bright sub-state is mostly decoupled from the anharmonic resonance. Characteristic of the anharmonic resonance, the oscillation frequency of the zeroth-order bright sub-state does not vary much with $J$. The rotational $l$-type resonance only contributes to fast oscillations between the two (01011) zeroth-order sub-states. The qualitative appearance of the survival probability at $J=100$ is similar to that for $J=30$ in Figure 2d, but the interactions involved are different.

The dilution factor provides a convenient way of quantifying the extent of mixing of the different bright states in this 3 -state system. First, we note that the extent of mixing may vary non-monotonically with $J$. For example, with the bright sub-state $(01011,1-1), \Sigma_{e u}$ (Fig. 3c), $\phi_{d}$ varies from 0.49 to 0.40 to 0.48 for $J=2,30$ and 100, respectively. The extent of mixing also depends on which basis state is identified as the bright state. For the infrared-active bright state, $(00100,00), \Sigma_{e u}$
Deleted: effects
Formatted: Don't adjust right indent when grid is defined, Space After: 0 pt, No widow/orphan control, Don't adjust space between Latin and Asian text, Don't adjust space between Asian text and numbers,

Tabs: Not at $0 \mathrm{pt}+18 \mathrm{pt}+$ $134.7 \mathrm{pt}$ 
(Fig. $4 \mathrm{~d}$ ), $\phi_{d}$ varies from 0.49 to 0.48 to 0.89 as $J$ increases. The large $\phi_{d}$ at $J=100$ reflects the decoupling of this bright state at high $J$. The practical way to modify the nature of the bright state is to exploit different spectroscopic techniques, such as using IR absorption or stimulated emission pumping (SEP). Thus when different spectroscopic techniques are used to prepare states in the same energy range of the same molecule, and even in the same polyad, one can expect to see different dilution factors and rather different time-dependent behavior. As a practical matter in acetylene, the $g / u$ selection rules prevent the IR and SEP techniques from reaching the same polyad, so a 3-photon variant of $\mathrm{SEP}_{-1}^{37}$ would be needed to reach the bright sub-state $(01011,1-1), \Sigma_{\text {eu }}$.

\section{Coriolis resonances}

As pointed out above, Coriolis resonances act as symmetry-breaking interactions. They mix even and odd $k$ blocks otherwise belonging to different polyads. As an example, we will use the same infrared-active zeroth-order bright sub-state $(00100,00), \Sigma_{\text {eu }}$ as in the previous example. With the Coriolis interaction included, the polyad size is now increased to 17, from the 3 in Figures 3 and 4 . A reduced energy graph of a selected, relevant subset of these 17 sub-states is represented in Figure 5a. Coriolis resonances are induced in the present case by the 2/444 coupling scheme, bringing in the polyad states such as (00041),
Deleted: change

Deleted: use

Deleted: $\{$ Hoshina, K.; Iwasaki A.; Yamanouchi, K.; Jacobson, M. P.; Field, R. W. J. Chem. Phys. 2001, 114, 7424-7442.\}

Formatted: Font: Century Schoolbook

Formatted: Font: (Default) Helvetica, Complex Script Font: Helvetica

Deleted: sub-states with
different
Deleted:
Deleted: -parity
Deleted: previously


interacting with (01011), with their various $k$-components. The Coriolis matrix elements are given by

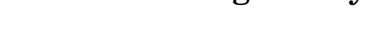

with $F_{ \pm}(J, k)=[J(J+1)-k(k \pm 1)]^{1 / 2}$.

Like the rotational $l$-type resonance, the Coriolis couplings depend explicitly on rotation. Although the value of the $\mathrm{C}_{2 / 444}$ parameter at $7.810^{-3} \mathrm{~cm}^{-1} \underline{43}$ is similar to Formatted: Superscript the size of the $q_{4}$ and $q_{5}$ parameters in the rotational $l$-type resonance, the rotational dependence is, however more modest, $\sim[J(J+1)]^{1 / 2}$ rather than $\sim J(J+1)$, resulting in much smaller coupling matrix elements, $\sim 0.5 \mathrm{~cm}^{-1}$ at $J=100$ as opposed to $\sim 50$ $\mathrm{cm}^{-1}$. Therefore, the Coriolis couplings appear mainly as local avoided crossings, affecting only few $J$ s. The avoided crossings can be easily identified in Figure $5 \mathrm{a}$ as the locations of abrupt changes in the slope of the eigenstate reduced-energy curves. The eigenstates in Figure 5a are labeled accounting for the avoided crossings using different marker styles with the lowest being I and the highest VII. If one did not include the Coriolis interactions giving rise to these local avoided crossings, plots of the eigenenergies versus $J$ would follow smooth curves without abrupt changes in slope. These smooth curves are evident in Fig. 5a but have an abrupt marker style change at each avoided crossing. They represent vibrational sub-states whose vibrational character varies only slowly with $J$, and is defined by the combined effects of the two strong interactions, the rotational $l$-type resonance and the 
anharmonic 2/345 resonance. For example, at low $J$, the eigenstates labeled V, VI and VII in Figure 5a are to a good approximation the same as the eigenstates labeled as I, II, and III respectively in Figure 4a. The eigenstate II in Figure 4a becomes the smooth curve in Figure 5a that begins as eigenstate VI at low $J$, and then becomes successively V, IV, and finally III at $J=100$.

The amount of the bright zeroth-order sub-state in eigenstates I to VII was calculated from the eigenvectors in the matrix diagonalisation process and is plotted in Figure 5b. The scheme bears some resemblance to Figure $4 \mathrm{~b}$ but is obviously more complicated. The curves of Figure $4 \mathrm{~b}$ become the smoothly varying curves in Figure 5b. As in Figure 5a, the abrupt changes in marker style along the smooth curves in Figure 5b indicate the locations of the Coriolis resonances.

To examine the effects of the Coriolis resonances, we will focus on a particular example, the avoided crossing in Figure 5 a at $J=92$, between eigenstates I ( $\square$ ) and II ( $\Delta$ ). The amount of the zeroth-order bright sub-state $(00100,00), \Sigma_{u}^{+}$, for the different $J_{\mathrm{s}}$ is quantified in Figure $5 \mathrm{~b}$. The resulting intensity borrowing in the vicinity of $J=92$ is illustrated in Figure 5c. Since the mixing of the two Corioliscoupled levels is maximum for $J=92$, one can expect an impact on the survival probability for this specific level. Time-dependent curves are presented in Figure 5d for $J=92$ (lower part), and also for a non-perturbed level, $J=88$ (upper part), for comparison._As already demonstrated, the strength of the Coriolis coupling is weak. Nonetheless, the various $k$-components of the (00041) zeroth-order state which are
Deleted: sub-

Deleted: sub-

Deleted: a diabatic state

Deleted: . It

Deleted: evolves along a smooth curve, becoming

Deleted: diabatic

Deleted: diabatic

Deleted:

Deleted: is 
Coriolis-coupled to (01011), do take part in the dynamical behavior at $J=92$. At $J=$

92, the total probability of the Coriolis-coupled states reaches a maximum of $\sim 10 \%$ at $8 \mathrm{ps}$ and proceeds to oscillate weakly with a period of $16 \mathrm{ps.} \mathrm{Although} \mathrm{there} \mathrm{are}$ numerous Coriolis resonances (Fig. 5a), and such resonances do affect the dynamics at the localized $J$ 's where they occur (Fig. 5d), most $J$ 's remain more-or-less unaffected.

\section{Thermally averaged dynamics}

In the previous section, we have explored the basic mechanisms perturbing the zeroth-order vibration-rotation picture in acetylene in the lower energy region $(0$ $-8,600 \mathrm{~cm}^{-1}$ ) where its rotation-vibration spectroscopy is most complete. The same mechanisms rule the time-dependent dynamics resulting from coherent excitations. Since all of the interactions that are significant in this energy region are known to good precision, the rate at which energy arrives in the receiving modes as well as the rate at which it leaves the bright state can be calculated reliably. That is, the complete vibrational dynamics are obtainable from the experimental Hamiltonian. The detailed rotational analysis in this region allowed us to examine the rotational dependence of the dynamics. Since some of the important intramolecular interactions, such as the rotational $l$-type resonance and Coriolis interactions, 
increase in strength with rotational angular momentum, there are significant changes in the pattern of the dynamics with rotational excitation as has been demonstrated above. In the present section, we shall examine the results of exciting a thermal distribution of rotational levels. In this case, a blend of the basic mechanisms just highlighted is involved._ The temperature regulates the rotational contribution in the dynamics. The population distributions in the GS are presented for the three selected temperatures, 27, 270 and $500 \mathrm{~K}$, in Figure 6. The behavior is also expected to depend strongly on the polyad and on zeroth-order bright substate. Polyads $\{7, e, u\}$ and $\{7, e, g\}$ were selected as working examples, both near 4500 $\mathrm{cm}^{-1}$ and therefore in the spectroscopically well characterized energy region. All of the interaction mechanisms contained in the global Hamiltonian are included in the calculations. For the two case studies, the GS rotational distribution is simply projected onto the $e$-levels of the upper zeroth-order bright sub-state. We did not include the detailed rotational line strengths or the particular rotational selection rules appropriate to a given spectroscopic technique. As a result, rotational coherences ${ }^{67}$ have been suppressed. However, we did include the appropriate nuclear spin weights appropriate: 1:3 for even:odd $J$ for sub-states in $\{7, e, g\}$ and 3:1 in $\{7, e, u\}$ (see Figure 27 in Herman ${ }_{4}^{24}$ ). This means that a coherent excitation with an infrared source, which produces $\underline{u}$ upper states from the $g$ GS, would yield a preponderance of even $J$ upper state molecules. Likewise a coherent Raman or SEP excitation would yield odd $J$ 's preferentially (if proceeding through R- or P- line excitation). This rule applies, in both cases, for $e$-symmetry levels.
Deleted: around
Formatted: Superscript

Deleted:

Formatted: Superscript

Deleted: ${ }^{24}$

Deleted: broadband

Deleted: broadband 


\section{A. Polyad $\{7, e, u\}$}

The zeroth-order IR bright sub-state in the $\{7, e, u\}$ polyad is $(00120,00), \Sigma_{e u}$. The complex set of global and local perturbations is evident from the irregular $J$ dependence of the reduced energies in Figure 7a. All of the interactions are well characterized spectroscopically $\frac{43}{49}$ The contribution of the bright zeroth-order subFormatted: Superscript Deleted: ${ }^{43}$ state to the selected 10 relevant eigenstates, labeled I to X, is shown in Figure $7 \mathrm{~b}$. Since there are a total of 45 sub_states in the polyad, each of the parts of Figure 7 are simplified to show only the most significant quantities.

The dynamics is presented in Figure 7c. Thermal averaging obviously smoothes out the detailed interaction pattern. Nevertheless, one can identify the main trends of the behavior in terms of the basic intramolecular couplings investigated in the previous section. At low temperature, since only the very first $J$ levels are populated, the dynamics is dominated by the $3 / 245$ anharmonic resonance connecting the $(00120,00)$ and $(01031,1-1)$ sub-states. The probability oscillates between these two sub_states with a period of $1.7 \mathrm{ps.} \mathrm{This} \mathrm{is} \mathrm{the} \mathrm{pattern} \mathrm{expected}$ for a 2-state system (Eq. (5)) with little involvement from other states in the polyad. At 270 and $500 \mathrm{~K}$, higher $J$-levels are involved and the rotational $l$-type resonance plays an increasing role. The rotational $l$-type resonance-coupled sub-state (01031,3-1) reaches a maximum probability after about $3 \mathrm{ps,}$, and oscillations ensue 
with a 7 ps period. This sub-state in turn resonates anharmonically with $(00120,20)$ and proceeds to oscillate with it on a $1.7 \mathrm{ps}$ period.

At 270 and $500 \mathrm{~K}$, the oscillations in Figure 7c appear to damp out at later times. The rotational $l$-type resonance oscillations damp out so quickly that only one complete oscillation is discernable in Figure 7c. The anharmonic oscillations damp more slowly with a timescale of roughly 100 ps. At first, it may seem surprising that there is any damping of the oscillations since Figure 7c represents collisionless dynamics in the very sparse limit, with $\phi_{d}=0.49,0.43$, and 0.39 for $\mathrm{T}=$ 27,270 , and $500 \mathrm{~K}$, respectively. There is no coupling to any kind of external bath included in the model Hamiltonian, and the oscillations resulting from the excitation of a single $J$ should continue indefinitely. The damping apparent in Figure $7 \mathrm{c}$ results from the dephasing of the oscillations of different $J$-levels that have different oscillation frequencies. Indeed, in their early ultrafast experiments, Felker and Zewail $\frac{68}{4}$ noted the damping of oscillations by small frequency Formatted: Superscript Deleted: ${ }^{67}$ differences among the thermally-averaged rotational levels. In the present case, the dephasing of the rotational $l$-type resonance oscillations is rapid because of the explicit $J$-dependence of the coupling (Eq. (12)). The anharmonic resonance Deleted: 11 oscillations damp out much more slowly because the anharmonic interaction has only the incidental rotational dependence that results from the slight variation of the rotational constants with different kinds of vibrational excitation. Thus, the apparently irreversible decay of the oscillations in Figure $7 \mathrm{c}$ does not mean that any 
1

2

3

4

5

6

7

8

9 individual rotational level decays irreversibly, but it is an artifact of the inhomogeneous rotational structure within the coherent excitation bandwidth.

Although Coriolis coupling is involved at intermediate $J$-values in the complex interaction pattern shown in Figure 7a, it remains very local and therefore does not play a significant role in the averaged dynamics.

\section{B. Polyad $\{7, e, g\}$}

There is no IR allowed transition from the GS to any state in the $\{7, e, g\}$ polyad, all of which have g-symmetry. The SEP bright sub-states are $(01040,00), \Sigma_{\text {eg }}$ and $(01040,20), \Delta_{\mathrm{g}}$. Richer rotationally-dependent dynamics can, however, be expected from the $(00070,10), \Pi_{e g}$ sub-state in this polyad. This sub-state is indeed mixed through the 44/55 anharmonic resonance to other sub-states such as (00052). In addition, rotational $l$-type resonance generates strong couplings for this sub-state spanning $k$-values from 1 to 7 , by steps of 2 . Furthermore, it is coupled to the previously identified SEP bright sub-states thanks to 2/444 Coriolis resonance. We have therefore selected it as our hypothetical zeroth-order bright sub-state. The reduced energy graph is presented in Figure 8a, with only relevant zeroth-order and eigenstates presented. The corresponding sub-state fractionation and dynamics are 
The time-dependent dynamics in Figure 8c look quite different from the previous case (Fig. 7c). At low temperature, the behavior is dominated by the $44 / 55$ anharmonic resonance. It is too far off resonance to generate much redistribution, but it does result in high-frequency ( $0.2 \mathrm{ps})$, small amplitude (12\%) oscillations in the zeroth-order bright sub-state survival probability. _These small oscillations persist at the higher temperatures and out to long times. At the higher temperatures, the $J$-dependent interactions get stronger and induce what appears to be a rapid irreversible decay of the bright-state survival probability, $\tau_{\mathrm{IVR}}=1.3$ and $0.9 \mathrm{ps}$ for 270 and $500 \mathrm{~K}$, respectively. The absence of substantial recurrences results from the rotationally-dependent intramolecular couplings highlighted above.

Both rotational $l$-type resonance, bringing in the other sub-states from (00070), and Coriolis coupling, bringing in the (01040) sub-states play an active role in the energy redistribution. The rotational l-type resonance within (01040) further fractionates the redistribution.

Insight into the nature of the apparent irreversible decays in Figure 8c can be obtained by examining the extent of mixing of the zeroth-order bright sub-state as measured by the dilution factor. We find $\phi_{d}=0.84,0.51,0.43$ for the temperatures 27,270 and $500 \mathrm{~K}$ respectively, which indicates that the fractionation for this $g$ symmetry state is comparable to but slightly less than for the $u$-, IR-active bright state represented in Figure 7. Just as for the above case (Fig. 7c), each of the constituent rotational levels contributing to Figure 8c will exhibit substantial
Deleted: is observed for the first time in the selected case studies. It

Deleted: entirely from the basic Deleted: of rotational origin

Deleted: Rotational
Deleted:

Deleted: like 
recurrences extending out to long times (similar to Figs. 2d and 4d). The curves in Figure $8 \mathrm{c}$ are the thermal average of curves with differing oscillation frequencies. In Figure 7 , the strongest mixing of the basis states is caused by the anharmonic resonance (large fast oscillations in Fig. 7c), which is only indirectly $J$-dependent. However in Figure 8, this interaction results in less mixing (small fast oscillations in Fig. 8c), and the dominant mixing comes from the $J$-dependent rotational $l$-type resonance. This gives rise to a stronger $J$-dependence of the constituent oscillation frequencies in Figure 8c, and the thermal average of such a wide range of oscillation frequencies has the appearance of an irreversible decay. Once again, this is inhomogeneous dynamics resulting from the inhomogeneous rotational structure within the coherent excitation bandwidth.

\section{Conclusion}

The connection between the energy-resolved spectra and time-resolved dynamics has been explored in acetylene $\left({ }^{12} \mathrm{C}_{2} \mathrm{H}_{2}\right), \tilde{X}^{1} \Sigma_{g}^{+}$in a way that complements previous literature results ${ }_{4,26,30,35,36,37}^{25,}$ Here, the emphasis is on the impact of $]$ rotational excitation on the time-dependent dynamics, which has not been considered in detail in the previous acetylene literature. The present results derive from the extensive and reliable knowledge of the vibration-rotation energy levels

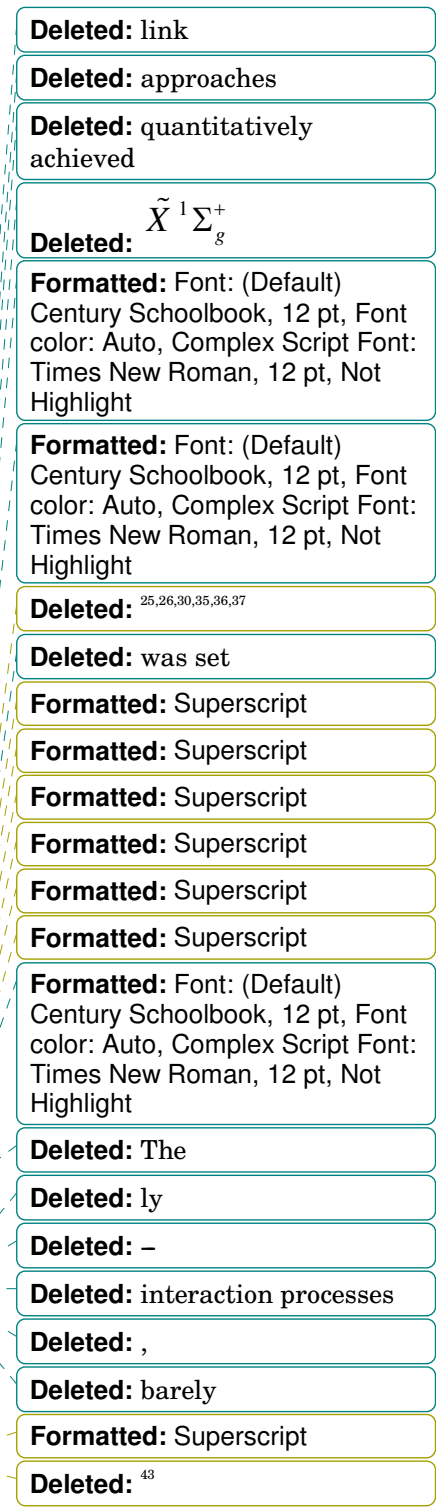


and from a global spectroscopic Hamiltonian based on the block diagonalisation of vibrational polyads. Three kinds of intramolecular interactions, anharmonic, rotational $l$ - and Coriolis resonances, perturb the zeroth-order vibration-rotation picture. The roles of each were quantitatively investigated for selected case studies well within the range of the fitted spectroscopic data $\left(0-8,600 \mathrm{~cm}^{-1}\right)$. The fractionation of each bright sub-state into the various eigenstates of its polyad was demonstrated to affect both the intensities in the spectra and the IVR survival probabilities. The collisionless dynamics resulting from the excitation of thermal distributions of rotational levels was investigated for the $v_{3}+2 v_{4}$ and $7 v_{4}$ bright states. The resulting inhomgeneous dynamics showed damped oscillatory survival probabilities for $v_{3}+2 v_{4}$ and apparent irreversible decay for $7 v_{4}$. It will be interesting to build on the present results to investigate the more extensive IVR that occurs at higher excitation energies,

Although actual time-dependent experiments haven't yet been performed on this system, the detailed spectroscopic Hamiltonian allows the results of a wide variety of virtual time-dependent experiments to be calculated. This kind of simulation enlightens key issues in time-dependent experiments. First, the identity of the vibrational bright state is critical in determining the time-dependent dynamics. Different bright states in the same polyad or same energy region produce very different results, whereas similar bright states tend to produce similar results over a range of energies. Second, in the case of overlapping polyads, virtual

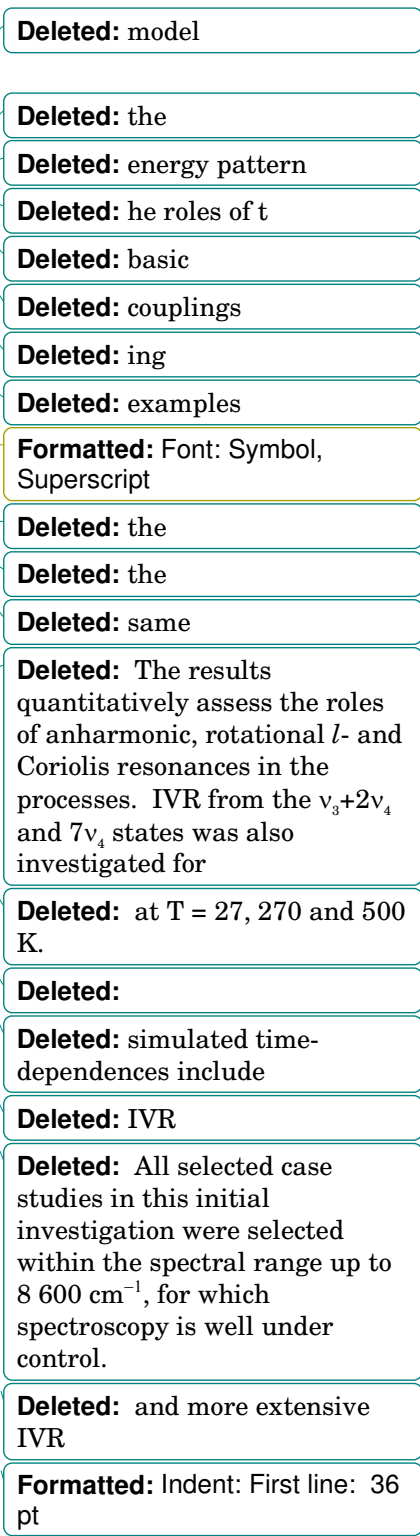


excitations can be performed on individual polyads separately, as opposed to the superposition of polyads that one might get in a practical experimental excitation. Finally, the effects of inhomogeneous broadening within the excited spectrum can be simulated, and these effects may change the qualitative form of the timedependent survival probabilities.

Formatted: English U.S.

Acknowledgements
The referee is thanked for a detailed commentary that has led to a clearer,
more insightful paper. This work was supported, in Brussels, by the Fonds
National de la Recherche Scientifique (F.R.S.-FNRS, contracts FRFC and IISN) and
the "Action de Recherches Concertées de la Communauté française de Belgique ".
The work was supported by the Division of Chemical Sciences, Offices of Basic
Energy Sciences, Office of Energy Research, U.S. Department of Energy under
Grant No. DE-FG02-90ER14151. Support of this work does not constitute
endorsement by the DOE of views expressed in this paper._B.A. thanks FRIA for a
research grant.

Deleted: the various comments
Deleted: which
Deleted: helped improving the
pedagogical
Deleted: in the
Formatted: French Belgium




\section{References}

1 E. Abramson, R. W. Field, D. Imre, K. K. Innes, and J. L. Kinsey, J. Chem. Phys. 80, 2298 (1984).

2 A. M. de Souza, D. Kaur, and D. S. Perry, J. Chem. Phys. 88, 4569 (1988).

3 K. K. Lehmann, B. H. Pate, and G. Scoles, Annu. Rev. Phys. Chem. 45, 241 (1994).

$4 \quad$ D. J. Nesbitt and R. W. Field, J. Phys. Chem. 100, 12735 (1996).

5 J. D. McDonald, Annu. Rev. Phys. Chem. 30, 29 (1979).

6 J. Manz and L. Wöste, Femtosecond chemistry - Vol.1. (VCH, 1995).

7 A. A. Stuchebrukhov and R. A. Marcus, J. Chem. Phys. 98, 6044 (1993).

8 D. Madsen, R. Pearman, and M. Gruebele, J. Chem. Phys. 106 (14), 5874 (1997).

$9 \quad$ E. Fermi, Z. Phys. 71, 250 (1931).

10 B. T. Darling and D. M. Dennison, Phys. Rev. 57, 128 (1940).

11 A. Mehta, A. A. Stuchebrukhov, and R. A. Marcus, J. Phys. Chem. 99, 2677 (1995).

12 R. Pearman and M. Gruebele, J. Chem. Phys. 108 (16), 6561 (1998).

13 L. Lubich, O. V. Boyarkin, R. D. F. Settle, D. S. Perry, and T. R. Rizzo, Faraday Discussions 102 (Unimolecular Reaction Dynamics), 167 (1996).

14 O. V. Boyarkin, T. R. Rizzo, and D. S. Perry, J. Chem. Phys. 110 (23), 11346 (1999).

15 M. Gruebele, Proc. Natl. Acad. Sci. U. S. A. 95 (11), 5965 (1998).

16 M. Gruebele and V. Wong, Adv. Multi-Photon Processes Spectrosc. 14 (Quantum Control of Molecular Reaction Dynamics), 47 (2001).

17 R. Bigwood and M. Gruebele, Chem. Phys. Lett. 235 (5,6), 604 (1995).

18 M. Gruebele, J. Phys. Chem. 100 (30), 12183 (1996).

19 J. Go and D. S. Perry, J. Chem. Phys. 103 (13), 5194 (1995). 
20 D. S. Perry, G. A. Bethardy, M. J. Davis, and J. Go, Faraday Discussions 102 (Unimolecular Reaction Dynamics), 215 (1996).

21 A. Campargue, L. Biennier, A. Garnache, A. Kachanov, D. Romanini, and M. Herman, J. Chem. Phys. 111, 7888 (1999).

22 M. Herman, J. Liévin, J. Vander Auwera, and A. Campargue, Adv. Chem. Phys. 108, 1 (1999).

23 V. I. Perevalov, S. A. Tashkun, O. M. Lyulin, and J.-L. Teffo, The NATO Programme for Security through Science C: Environmental security (Springer), 139 (2006).

24 M. Herman, Mol. Phys. 105, 2217 (2007).

25 M. I. El Idrissi, B. Zhilinskii, P. P. Gaspard, and M. Herman, Mol. Phys. 101, 595 (2003).

26 M. Abbouti Temsamani, M. Herman, S. A. B. Solina, J. P. O'Brien, and R. W. Field, J. Chem. Phys. 105, 11357 (1996).

27 M. P. Jacobson, J. P. O'Brien, R. J. Silbey, and R. W. Field, J. Chem. Phys. 109, 121 (1998).

28 M. P. Jacobson, J. P. O'Brien, and R. W. Field, J. Chem. Phys. 109, 3831 (1998).

29 M. P. Jacobson, C. Jung, H. S. Taylor, and R. W. Field, J. Chem. Phys. 111, 600 (1999).

30 M. P. Jacobson and R. W. Field, J. Phys Chem. A 104, 3073 (2000).

31 J. P. Rose and M. E. Kellman, J. Chem. Phys. 105, 10743 (1996).

32 M. P. Jacobson, R. J. Silbey, and R. W. Field, J. Chem. Phys. 110, 845 (1999).

33 B. M. Wong, A. H. Steeves, and R. W. Field, J. Phys. Chem. B 110, 18912 (2006).

34 V. Tyng and M. E. Kellman, J. Phys. Chem. B, 18859 (2006).

35 D. M. Jonas, S. A. B. Solina, B. Rajaram, S. J. Cohen, R. J. Silbey, R. W. Field, K. Yamanouchi, and S. Tsuchiya, J. Chem. Phys. 99, 7350 (1993).

36 H. K. Srivastava, A. Conjusteau, H. Mabuchi, A. Callegari, K. K. Lehmann, G. Scoles, M. L. Silva, and R. W. Field, J. Chem. Phys. 113, 7376 (2000). 
37 K. Hoshina, A. Iwasaki, K. Yamanouchi, M. P. Jacobson, and R. W. Field, J. Chem. Phys. 114, 7424 (2001).

38 D. A. Dolson, K. W. Holtzclaw, S. H. Lee, S. Munchak, C. S. Parmenter, and B. M. Stone, Laser Chem. 2, 271 (1983).

39 H. Saigusa, B. E. Forch, and E. C. Lim, J. Chem. Phys. 78, 2795 (1983).

40 W. D. Lawrance and A. E. W. Knight, J. Phys. Chem. 92 (21), 5900 (1988).

41 A. E. W. Knight, Excited States, edited by E.C._Lim and K.K. Innes (Academic, New York) 7, 64 (1988).

42 A. Mcllroy, D. J. Nesbitt, E. R. T. Kerstel, B. H. Pate, K. K. Lehmann, and G. Scoles, J. Chem. Phys. 100, 2596 (1994).

43 B. Amyay, S. Robert, M. Herman, A. Fayt, B. Raghavendra, A. Moudens, J. Thiévin, B. Rowe, and R. Georges, J. Chem. Phys. 131, 114301 (2009).

44 L.-H. Xu, X. Wang, T. J. Cronin, D. S. Perry, G. T. Fraser, and A. S. Pine, J. Mol. Spectrosc. 185, 158 (1997).

45 M. Bixon and J. Jortner, J. Chem. Phys. 48, 715 (1968).

46 M. Bixon and J. Jortner, J. Chem. Phys. 50, 3284 (1969).

47 M. Bixon and J. Jortner, J. Chem. Phys. 50, 4061 (1969).

48 K. Freed, Topics Appl. Physics 15, 23 (1976).

49 A. E. Douglas, J. Chem. Phys. 45, 1007 (1966).

50 G. M. Stewart and J. D. McDonald, J. Chem. Phys. 78, 3907 (1983).

51 G. A. Bethardy, X. Wang, and D. S. Perry, Can. J. Chem. 72 (3), 652 (1994).

52 J. M. Brown, J. T. Hougen, K. P. Huber, J. W. C. Johns, I. Kopp, H. Lefebvre-Brion, A. J. Merer, D. A. Ramsay, J. Rostas, and R. N. Zare, J. Mol. Spectrosc. 55, 500 (1975).

53 M. Herman, Handbook of High Resolution Spectroscopy, Wiley M. Quack and F. Merkt Eds., in press (2009). 
54 M. E. Kellman, J. Chem. Phys. 93, 6630 (1990).

55 M. E. Kellman and G. Chen, J. Chem. Phys. 95, 8671 (1991).

56 M. E. Kellman, Annu. Rev. Phys. Chem. 46, 395 (1995).

57 C. Vigouroux, A. Fayt, A. Guarnieri, A. Huckauf, H. Burger, D. Lentz, and D. Preugschat, J. Mol. Spectrosc. 202, 1 (2000).

58 A. Fayt, S. Robert, C. Di Lonardo, L. Fusina, F. Tamassia, and M. Herman, J. Chem. Phys. 126, 114303 (2007).

59 D. B. Moss, Z. Duan, M. P. Jacobson, J. P. O'Brien, and R. W. Field, J. Mol. Spectrosc. 199, 265 (2000).

60 G. Amat and H. H. Nielsen, J. Mol. Spectrosc. 2, 152 (1958).

61 G. Amat and H. H. Nielsen, J. Mol. Spectrosc. 2, 163 (1958).

62 H. H. Nielsen, Rev. Mod. Phys. 23, 90 (1951).

63 H. H. Nielsen, Handb. Phys. 37/1, 173 (1959).

64 J. Plíva, J. Mol. Spectrosc. 44, 165 (1972).

65 J. K. G. Watson, J. Mol. Spectrosc. 101, 83 (1983).

66 W. J. Lafferty and R. J. Thibault, J. Mol. Spectrosc. 14, 79 (1964).

67 P. M. Felker, J. Phys Chem. A 96, 7844 (1992).

68 P. M. Felker and A. H. Zewail, J. Chem. Phys. 82 (7), 2994 (1985).

Figure legends

Figure 1: Basic scheme for IVR and intensity borrowing issues defining zeroth- 
order states, $\psi_{1}{ }^{0}$ and $\psi_{2}{ }^{0}$, and eigenstates, $\psi^{\mathrm{II}}$ and $\psi^{\mathrm{I}}$. The red/grey color code is selected to highlight the fraction of each zeroth-order wavefunction in the eigen wavefunctions.

Figure 2: Illustration of the effects of the rotational $l$-type resonance in ${ }^{12} \mathrm{C}_{2} \mathrm{H}_{2}$, $\tilde{X}^{1} \Sigma_{g}^{+}$, selecting the $\left\{0,2, k_{e}, e, u\right\}$ polyad with the bright state taken to be the $(00011,1-1), \Sigma_{\text {eu }}$ zeroth-order sub-state:

-(a) Reduced energy graph as a function of $J(J+1)$. The eigenstates (black) are labeled I and II according to the ordering of their energies. The zeroth-order substates are colored and identified on the left using $\left(v_{1} v_{2} v_{3} v_{4} v_{5}, l_{4} l_{5} e u\right)$;

Deleted: Reduced energies correspond to $E_{v r}-1.180155 J(J+1)+1$.

Deleted: sub-

Deleted: subeigenstates plotted as a function of $J$. The numbering and line marker styles in parts (a) and (b) are related to the eigenstate label and internally consistent. The zeroth-order bright sub-state is indicated as $\left(v_{1} v_{2} v_{3} v_{4} v_{5}, l_{4} l_{5} \mathrm{e} / \mathrm{f} \mathrm{g} / \mathrm{u}\right)$;

-(c) Calculated relative intensities of the $R$-branch lines in the I $\leftarrow$ GS and II $\leftarrow$ GS IR absorption bands. The simulated intensities are given in terms of the upper $J$ quantum number $\left(J^{\prime}\right)$ for a temperature of $500 \mathrm{~K}$. The $3: 1$ intensity alternation due 
to ortho and para acetylene has been cancelled by multiplying the lower intensity lines by a factor 3 ;

-(d) Calculated time-dependent probability of the basis states for $J=2$ (upper), 30 (medium) and 100 (lower). At $\mathrm{t}=0$, the bright state (red) begins at $P_{1}(t)=1.0$. The color scheme distinguishes the zeroth-order sub-states according to order of their appearance in the time evolution. The color and marker styles for the zeroth-order sub-states are the same in parts (a) and (d).

Figure 3: Illustration of the effects of the rotational $l$-type resonance combined with the $3 / 245$ anharmonic resonance in ${ }^{12} \mathrm{C}_{2} \mathrm{H}_{2}, \tilde{X}^{1} \Sigma_{g}^{+}$, selecting the $\left\{1,5, k_{e}, e, u\right\}$ polyad with the bright state taken to be the $(01011,1-1), \Sigma_{\text {eu }}$ zeroth-order sub-state:

-(a) Reduced energy graph of the sub-states in the polyad as a function of $J(J+1)$ The eigenstates (black) are labeled I to III according to their energies. The zerothorder sub-states are shown in color and identified on the left as $\left(v_{1} v_{2} v_{3} v_{4} v_{5}, l_{4} l_{5} e u\right)$;

-(b) Squared coefficients of the bright state in the composition of the eigenstates of the polyad, plotted as a function of $J$. The numbering and line marker styles in parts (a) and (b) are related to the eigenstate label and are internally consistent. The zeroth-order bright sub-state is indicated as $\left(v_{1} v_{2} v_{3} v_{4} v_{5}, l_{4} l_{5} \mathrm{e} / \mathrm{f} \mathrm{g} / \mathrm{u}\right)$;
Deleted: .

Deleted: Reduced energies correspond to $E_{v r}-1.173000 J(J+1)+1$.

Deleted: sub-

Deleted: also presented. They are

Deleted: colored

Deleted: sub- 
-(c) Calculated time-dependent probability of the basis states for $J=2$ (upper), 30 (medium) and 100 (lower). At $\mathrm{t}=0$, the bright state (red) begins at $P_{1}(t)=1.0$. The color scheme distinguishes the zeroth-order sub-states according to order of their appearance in the time evolution. The color and marker styles for the zeroth-order sub-states are the same in parts (a) and (c).

Figure 4: Illustration of the effects of the rotational $l$-type resonance combined to the $3 / 245$ anharmonic resonance in ${ }^{12} \mathrm{C}_{2} \mathrm{H}_{2}, \tilde{X}^{1} \Sigma_{g}^{+}$, selecting the $\left\{1,5, k_{e}, e, u\right\}$ polyad with the bright state taken to be the $(00100,00), \Sigma_{e u}$ zeroth-order sub-state:

\footnotetext{
-(a) Reduced energy graph of the sub-states in the polyad as a function of $J(J+1)$, The eigen states (black) are labeled I to III according to the ordering of the energy of the corresponding eigenvalues. The zeroth-order sub-states are colored and identified on the left as $\left(v_{1} v_{2} v_{3} v_{4} v_{5}, l_{4} l_{5} e u\right)$;
}

-(b) Squared coefficients of the bright state in the composition of the eigenstates of the polyad, plotted as a function of $J$. The numbering and line marker styles in parts (a) and (b) are related to the eigenstate label and internally consistent. The zeroth-order bright sub-state is indicated as $\left(v_{1} v_{2} v_{3} v_{4} v_{5}, l_{4} l_{5} \mathrm{e} / \mathrm{f} \mathrm{g} / \mathrm{u}\right)$;

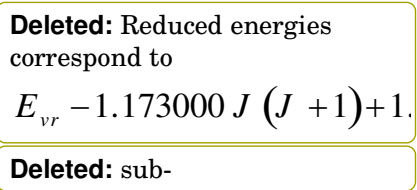

Deleted: sub- 
-(c) Calculated relative intensities of the $R$-branch lines in the I, II and III $\leftarrow$ GS IR absorption bands. The simulated intensities are given in terms of the upper $J$ quantum number $\left(J^{\prime}\right)$ for a temperature of $500 \mathrm{~K}$. The 3:1 intensity alternation due to ortho and para acetylene has been cancelled by multiplying the lower intensity lines by a factor 3 ;

-(d) Calculated time-dependent probability of the basis states for $J=2$ (upper), 30 (medium) and 100 (lower). At $\mathrm{t}=0$, the bright state (red) begins at $P_{1}(t)=1.0$. The color scheme distinguishes the zeroth-order sub-states according to order of their appearance in the time evolution. The color and marker styles for the zeroth-order sub-states are the same in parts (a) and (d).

Figure 5: Illustration of the effects of the 2/444 Coriolis resonance combined to rotational $l$-type resonance and $3 / 245$ anharmonic resonance in ${ }^{12} \mathrm{C}_{2} \mathrm{H}_{2}, \quad \tilde{X}^{1} \Sigma_{g}^{+}$, selecting the $\{5, e, u\}$ polyad with the bright state taken to be the $(00100,00), \Sigma_{e u}$ zeroth-order sub-state:

-(a) Reduced energy graph of selected sub-states in the polyad as a function of $J(J+1)$. The eigen states are identified by the marker style (black) and are labeled I to VII according to their energies. Only the four most relevant zeroth-order substates are colored and identified on the left as $\left(v_{1} v_{2} v_{3} v_{4} v_{5}, l_{4} l_{5} e u\right)$;
Deleted: Reduced energies correspond to $E_{v r}-1.173000 J(J+1)+1$. 
-(b) Squared coefficients of the eigenvector in the composition of the eigenstates in

the polyad, as a function of $J$. The numbering and line marker styles in parts (a) and (b) are related to the eigenstate label and internally consistent. The zerothorder bright sub-state is indicated as $\left(v_{1} v_{2} v_{3} v_{4} v_{5}, l_{4} l_{5} \mathrm{e} / \mathrm{f} \mathrm{g} / \mathrm{u}\right)$;

-(c) Resulting intensity borrowing on related $R$-branch lines simulated at a temperature of $2,000 \mathrm{~K}$, in function of the upper $J$ quantum number $\left(J^{\prime}\right)$. The two major contributions to the intensity are summed up, defining the grey bar;

-(d) Calculated time-dependent probability of the basis states for the Coriolis perturbed $J=92$ level (lower), compared $J=88$ level (upper), which is not Coriolisperturbed. Portions of the graphs shown on an expanded time and probability scales. The color and marker styles for the zeroth-order sub-states are the same in parts (a) and (d).

Figure 6: Population distribution in the rotational levels of the GS in ${ }^{12} \mathrm{C}_{2} \mathrm{H}_{2}, \tilde{X}^{1} \Sigma_{g}^{+}$, at 27,270 and $500 \mathrm{~K}$. The ortho and para distributions are color coded. 
Figure 7: The $\{7, e, u\}$ polyad in ${ }^{12} \mathrm{C}_{2} \mathrm{H}_{2}, \tilde{X}^{1} \Sigma_{g}^{+}$, with the zeroth-order bright sub-state taken to be $(00120,00), \Sigma_{e u}$ :

-(a) Reduced energy graph of selected sub-states as function of $J(J+1)$. The eigen states are identified by the marker style (black) and are labeled I to X according to their energies. Only the four most relevant zeroth-order sub-states are colored and identified on the left as $\left(v_{1} v_{2} v_{3} v_{4} v_{5}, l_{4} l_{5} e u\right)$;

-(b) Squared coefficients of the zeroth-order bright sub-state eigenvector in the composition of the eigenstates in the polyad, as a function of $J$. The numbering and line marker styles in parts (a) and (b) are related to the eigenstate label and are internally consistent. The zeroth-order bright sub-state is indicated as $\left(v_{1} v_{2} v_{3} v_{4} v_{5}, l_{4} l_{5}\right.$ $\mathrm{e} / \mathrm{f} \mathrm{g} / \mathrm{u})$

-(c) Calculated time-dependent probability of the basis states for a thermal averaged population in the upper zeroth-order bright sub-state, for $\mathrm{T}=27$ (upper), 270 (middle) and 500 (lower) K. The color code and line marker styles distinguish zeroth-order sub-states according to their successive rank in the time evolution. The color and marker styles for the zeroth-order sub-states are identical in parts (a) and (c).

Deleted: Reduced energies
correspond to
$E_{v r}-1.175076 J(J+1)+1$
Deleted: sub-
Deleted: The


Figure 8: The $\{7, e, g\}$ polyad in ${ }^{12} \mathrm{C}_{2} \mathrm{H}_{2}, \tilde{X}^{1} \Sigma_{g}^{+}$, with the zeroth-order bright sub-state taken to be $(00070,10), \Pi_{e g}$ :

-(a) Reduced energy graph of selected sub-states in function of $J(J+1)$. The eigen states are identified by the marker style (black) and are labeled I to VIII according to their energies. Only the five most relevant zeroth-order sub-states are colored and identified on the left as $\left(v_{1} v_{2} v_{3} v_{4} v_{5}, l_{4} l_{5} e u\right)$;

-(b) Squared coefficients of the $(00070,10), \Pi_{e g}$, zeroth-order sub-state eigenvector in the composition of the other eigenstates in the polyad, as a function of $J$. The Deleted: subnumbering and line marker styles in parts (a) and (b) are related to the eigenstate label and are internally consistent. The zeroth-order bright sub-state is indicated as $\left(v_{1} v_{2} v_{3} v_{4} v_{5}, l_{4} l_{5} \mathrm{e} / \mathrm{f} \mathrm{g} / \mathrm{u}\right)$

-(c) Calculated time-dependent probability of the basis states for a thermal averaged population in the upper zeroth-order bright sub-state, for $\mathrm{T}=27$ (upper), 270 (middle) and 500 (lower) K. The color scheme and line marker styles distinguish zeroth-order sub-states according to their order of appearance in the time evolution. The color and marker styles for the zeroth-order sub-states are the same in parts (a) and (c). 
Font: 16 pt, Italic

Page 4: [1] Formatted $\quad$ David Perry
Subscript

\begin{tabular}{|c|c|c|}
\hline Page 4: [2] Formatted & Mike & $1 / 25 / 201011: 37: 00$ AM \\
\hline
\end{tabular}

Page 4: [3] Formatted

Mike

1/25/2010 11:37:00 AM

Superscript

Page 4: [4] Formatted

Mike

1/25/2010 11:37:00 AM

Superscript

Page 4: [5] Formatted

Mike

1/25/2010 11:37:00 AM

Superscript

Page 4: [6] Deleted

Mike

1/25/2010 11:20:00 AM

Page 4: [6] Deleted Mike 1/20/2010 5:28:00 PM

In general, the notation $i j / m n$ indicates that quanta vibrational modes $i$ and $j$ involved are exchanged for quanta of modes $m$ and $n$.

$\begin{array}{lll}\text { Page 4: [7] Deleted } & \text { David Perry } & \text { 1/7/2010 5:38:00 PM }\end{array}$

\begin{tabular}{|c|c|c|}
\hline Page 4: [7] Deleted & David Perry & 1/7/2010 5:38:00 PM \\
\hline
\end{tabular}

URL: http://mc.manuscriptcentral.com/tandf/tmph 


\begin{tabular}{|c|c|c|}
\hline Page 4: [8] Formatted & Mike & 1/25/2010 11:37:00 AM \\
\hline \multicolumn{3}{|l|}{ Superscript } \\
\hline Page 4: [9] Formatted & Mike & 1/25/2010 11:37:00 AM \\
\hline \multicolumn{3}{|l|}{ Superscript } \\
\hline Page 4: [10] Formatted & Mike & 1/25/2010 11:37:00 AM \\
\hline \multicolumn{3}{|l|}{ Superscript } \\
\hline Page 4: [11] Formatted & Mike & 1/25/2010 11:37:00 AM \\
\hline \multicolumn{3}{|l|}{ Superscript } \\
\hline Page 4: [12] Formatted & Mike & 1/25/2010 11:37:00 AM \\
\hline Superscript & & \\
\hline
\end{tabular}

Page 4: [13] Formatted $\quad$ Mike
Superscript

Page 4: [14] Formatted $\quad$ Mike
Superscript

Page 4: [15] Formatted $\quad$ Mike
Superscript

Page 4: [16] Formatted Mike 1/25/2010 11:37:00 AM
Superscript

Page 4: [17] Formatted
Superscript

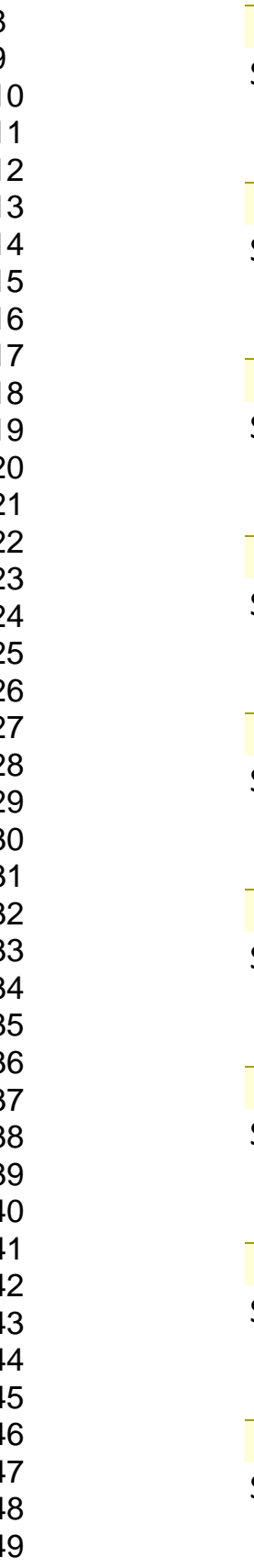



Superscript

Page 6: [26] Formatted Superscript

Page 6: [27] Formatted Superscript Superscript 


\section{Superscript}

Page 6: [32] Formatted

Mike

1/25/2010 11:37:00 AM

Superscript

\begin{tabular}{|c|c|c|}
\hline & Mike & 1/25/2010 11:37:00 AM \\
\hline & \multicolumn{2}{|c|}{ uperscript } \\
\hline Page 6: [34] Deleted & David Perry & 1/7/2010 9:02:00 PM \\
\hline
\end{tabular}
the bifurcation

\begin{tabular}{ccc}
$\begin{array}{c}\text { Page 6: [34] Deleted } \\
\text { of the normal modes }\end{array}$ & David Perry & 1/7/2010 9:00:00 PM \\
& & \\
\hline $\begin{array}{c}\text { Page 6: [34] Deleted } \\
\text { vibrational }\end{array}$ & David Perry & $1 / 7 / 2010$ 9:00:00 PM \\
\hline
\end{tabular}


Superscript

Page 6: [39] Formatted

Mike

1/25/2010 11:37:00 AM

Superscript

Page 6: [40] Formatted

Mike

1/25/2010 11:37:00 AM

Superscript

Page 6: [41] Formatted

Mike

1/25/2010 11:37:00 AM

Superscript

Page 6: [42] Formatted

Mike

1/25/2010 11:37:00 AM

Superscript

Page 6: [42] Formatted

Mike

1/25/2010 11:21:00 AM

Superscript

Page 6: [42] Formatted

Mike

1/25/2010 11:37:00 AM

Superscript

Page 6: [43] Formatted

Mike

1/25/2010 11:37:00 AM

Superscript

Page 6: [44] Formatted

Mike

1/25/2010 11:37:00 AM

Superscript

Page 6: [44] Formatted

Mike

1/25/2010 11:33:00 AM

Superscript

Page 6: [44] Formatted

Mike

1/25/2010 11:37:00 AM

Superscript

Page 6: [44] Formatted

Mike

1/25/2010 11:37:00 AM

Superscript

URL: http://mc.manuscriptcentral.com/tandf/tmph 


\begin{tabular}{|c|c|c|}
\hline Page 6: [45] Deleted & Mike & 1/25/2010 11:26:00 AM \\
\hline Page 6: [45] Deleted & Mike & 1/25/2010 11:06:00 AM \\
\hline Page 6: [46] Formatted & Mike & 1/25/2010 11:29:00 AM \\
\hline \multicolumn{3}{|l|}{ Superscript } \\
\hline Page 6: [46] Formatted & Mike & 1/25/2010 11:37:00 AM \\
\hline \multicolumn{3}{|l|}{ Superscript } \\
\hline Page 6: [47] Formatted & Mike & $1 / 25 / 201011: 37: 00$ AM \\
\hline Superscript & & \\
\hline
\end{tabular}

Superscript

URL: http://mc.manuscriptcentral.com/tandf/tmph 
Vibration-rotation alchemy in acetylene $\left({ }^{12} \mathrm{C}_{2} \mathrm{H}_{2}\right), \tilde{X}^{1} \Sigma_{g}^{+}$at low vibrational excitation: From high resolution spectroscopy to fast intramolecular dynamics

\section{To the publisher:}

This manuscript has 8 figures, most with multiple separately uploaded parts, which presents a special challenge for arranging the scaling the figure parts and for arranging the parts and captions on the journal page. Accordingly, we have supplied this document that show what the authors have in mind for the layout of the figures. Note that the figure parts with a time axis are shown larger on the page $(11-12 \mathrm{~cm}$ wide) than the other parts. When the figure parts are properly scaled in this way, the font sizes in all of the parts are about the same.

David Perry

dperry@uakron.edu

330-972-6825 


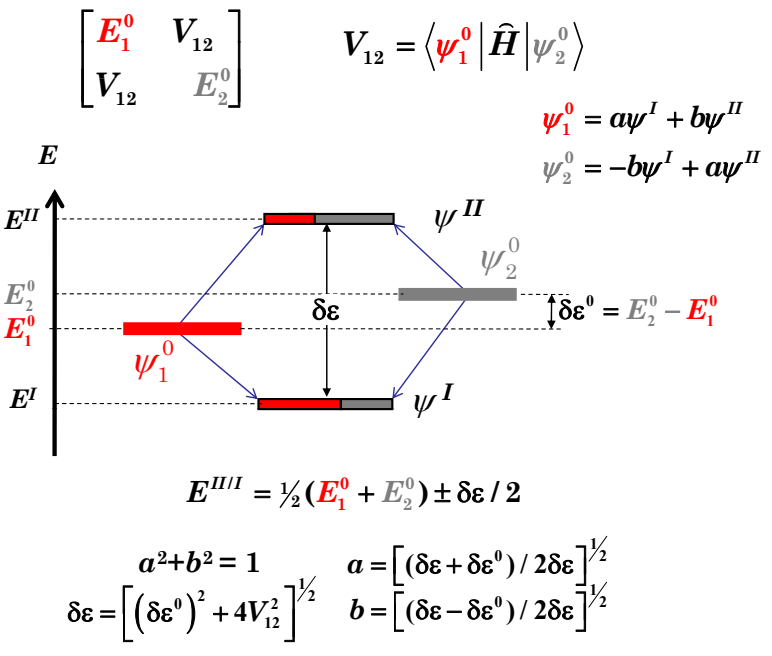

Figure 1: Basic scheme for IVR and intensity borrowing issues defining zeroth-order states, $\psi_{1}^{\circ}$ and $\psi_{2}^{\circ}$, and eigenstates, $\psi^{\mathrm{II}}$ and $\psi^{\mathrm{I}}$. The red/grey color code is selected to highlight the fraction of each zeroth-order wavefunction in the eigen wavefunctions. 
(a) $E_{V R}-1.180155 \mathrm{~J}(\mathrm{~J}+1)+1.626110^{-6} \mathrm{~J}^{2}(\mathrm{~J}+1)^{2}\left(\mathrm{~cm}^{-1}\right)$

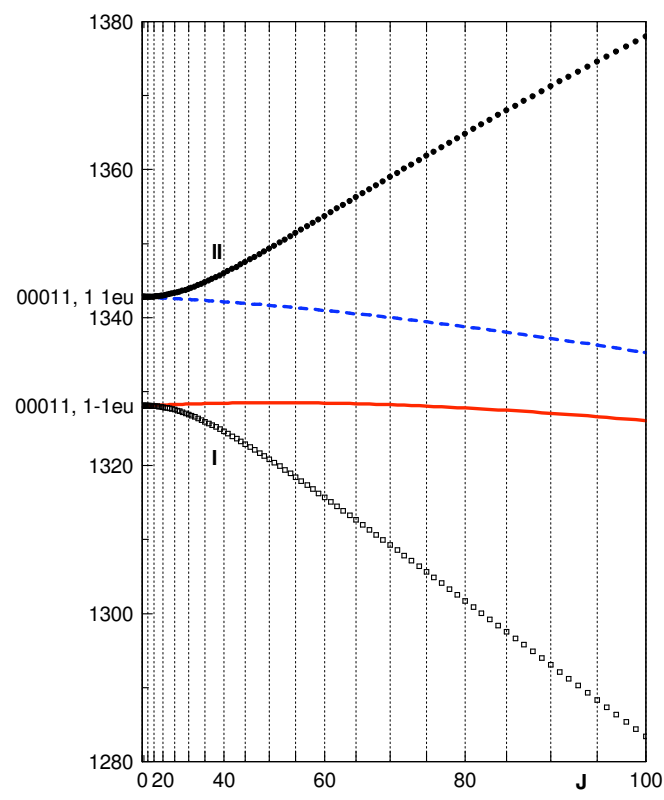

(b) Squared eigencoefficient value

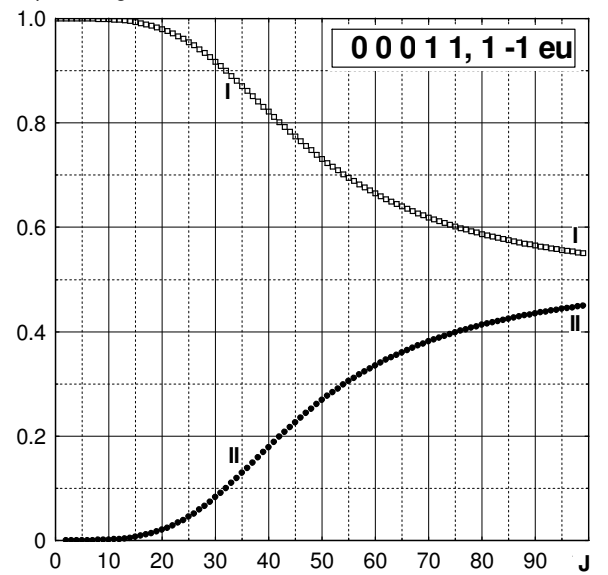

(c) Relative intensity

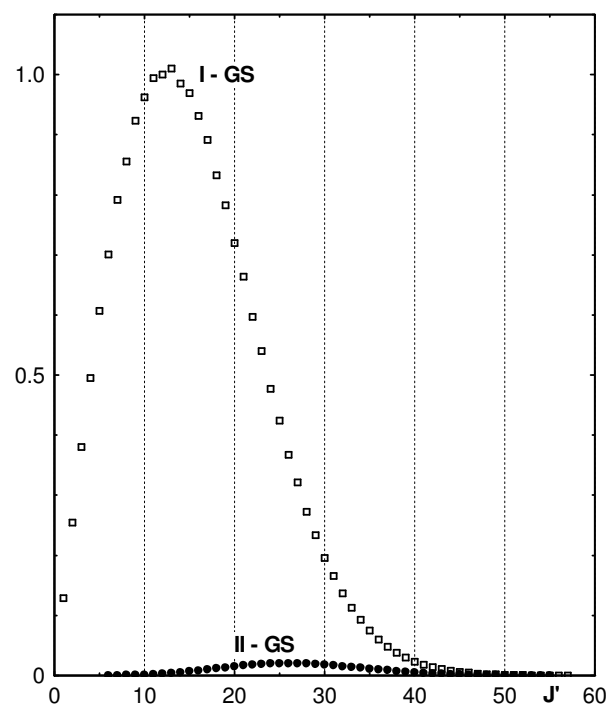

(d) $\mathrm{J}=2$
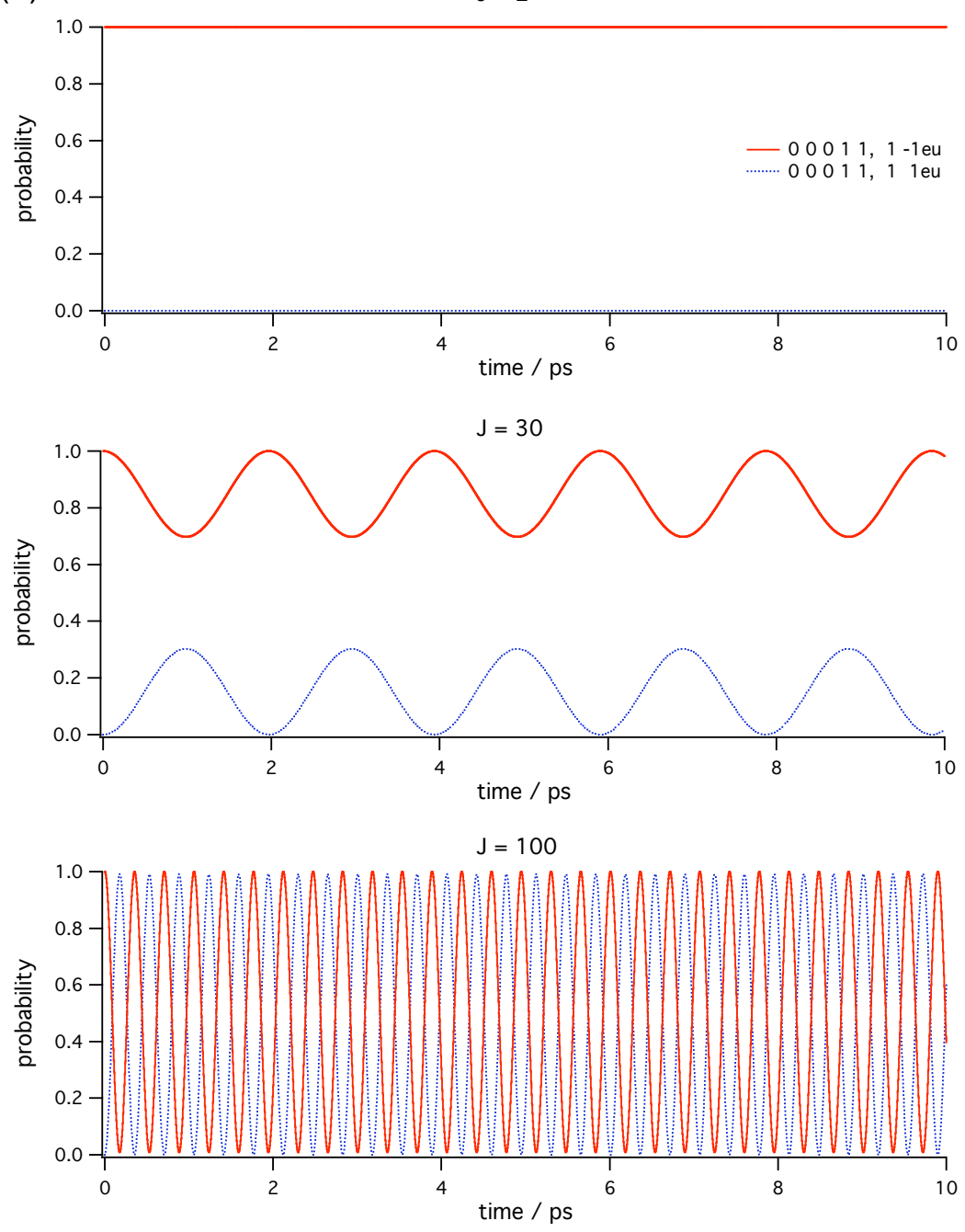

Figure 2: Illustration of the effects of the rotational $l$-type resonance in ${ }^{12} \mathrm{C}_{2} \mathrm{H}_{2}, \tilde{X}^{1} \Sigma_{g}^{+}$, selecting the $\left\{0,2, k_{e}, e, u\right\}$ polyad with the bright state taken to be the (00011,1-1), $\Sigma_{\text {eu }}$ zeroth-order sub-state:

(a) Reduced energy graph as a function of $J(J+1)$. The eigenstates (black) are labeled I and II according to the ordering of their energies. The zeroth-order sub-states are colored and identified on the left using $\left(v_{1} v_{2} v_{3} v_{4} v_{5}, l_{4} l_{5} e u\right)$;

(b) Squared coefficients of the bright state in the composition of the energy eigenstates plotted as a function of $J$. The numbering and line marker styles in parts (a) and (b) are related to the eigenstate label and internally consistent. The zeroth-order bright sub-state is indicated as $\left(v_{1} v_{2} v_{3} v_{4} v_{5}, l_{4} l_{5} \mathrm{e} / \mathrm{f} \mathrm{g} / \mathrm{u}\right)$;

(c) Calculated relative intensities of the $R$-branch lines in the $\mathrm{I} \leftarrow$ GS and II $\leftarrow$ GS IR absorption bands. The simulated intensities are given in terms of the upper $J$ quantum number $\left(J^{\prime}\right)$ for a temperature of $500 \mathrm{~K}$. The 3:1 intensity alternation due to ortho and para acetylene has been cancelled by multiplying the lower intensity lines by a factor 3 ;

(d) Calculated time-dependent probability of the basis states for $J$ $=2$ (upper), 30 (medium) and 100 (lower). At $\mathrm{t}=0$, the bright state (red) begins at $P_{1}(t)=1.0$. The color scheme distinguishes the zerothorder sub-states according to order of their appearance in the time evolution. The color and marker styles for the zeroth-order sub-states are the same in parts (a) and (d). 


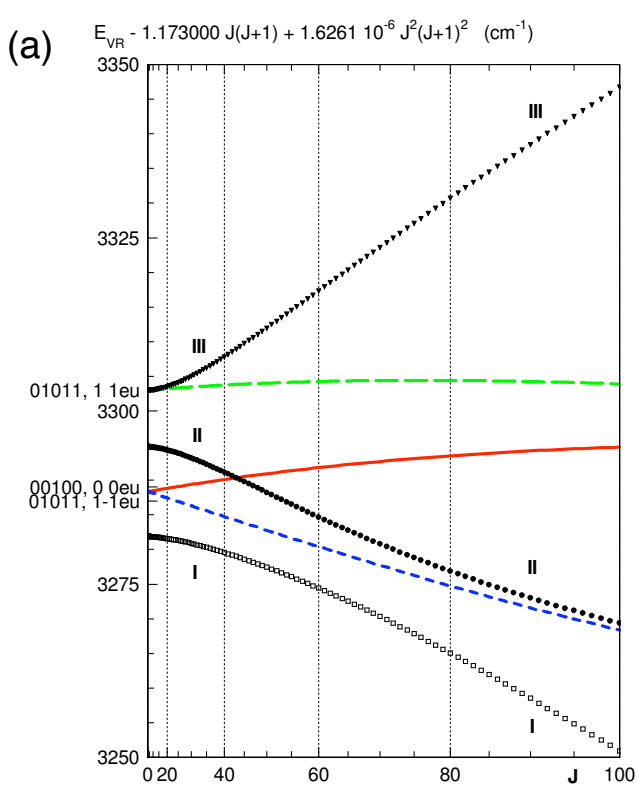

(b)

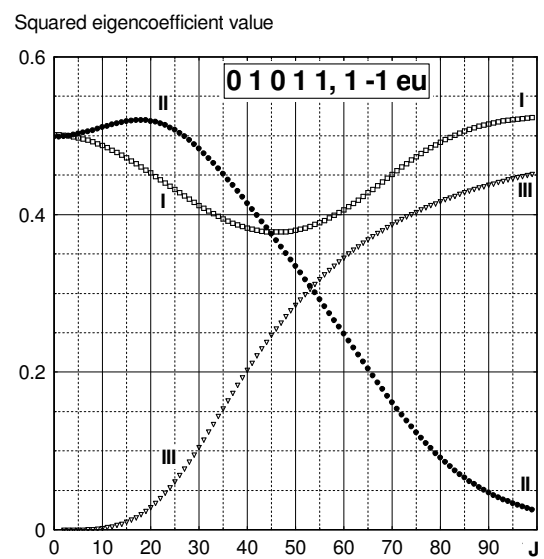

(c)
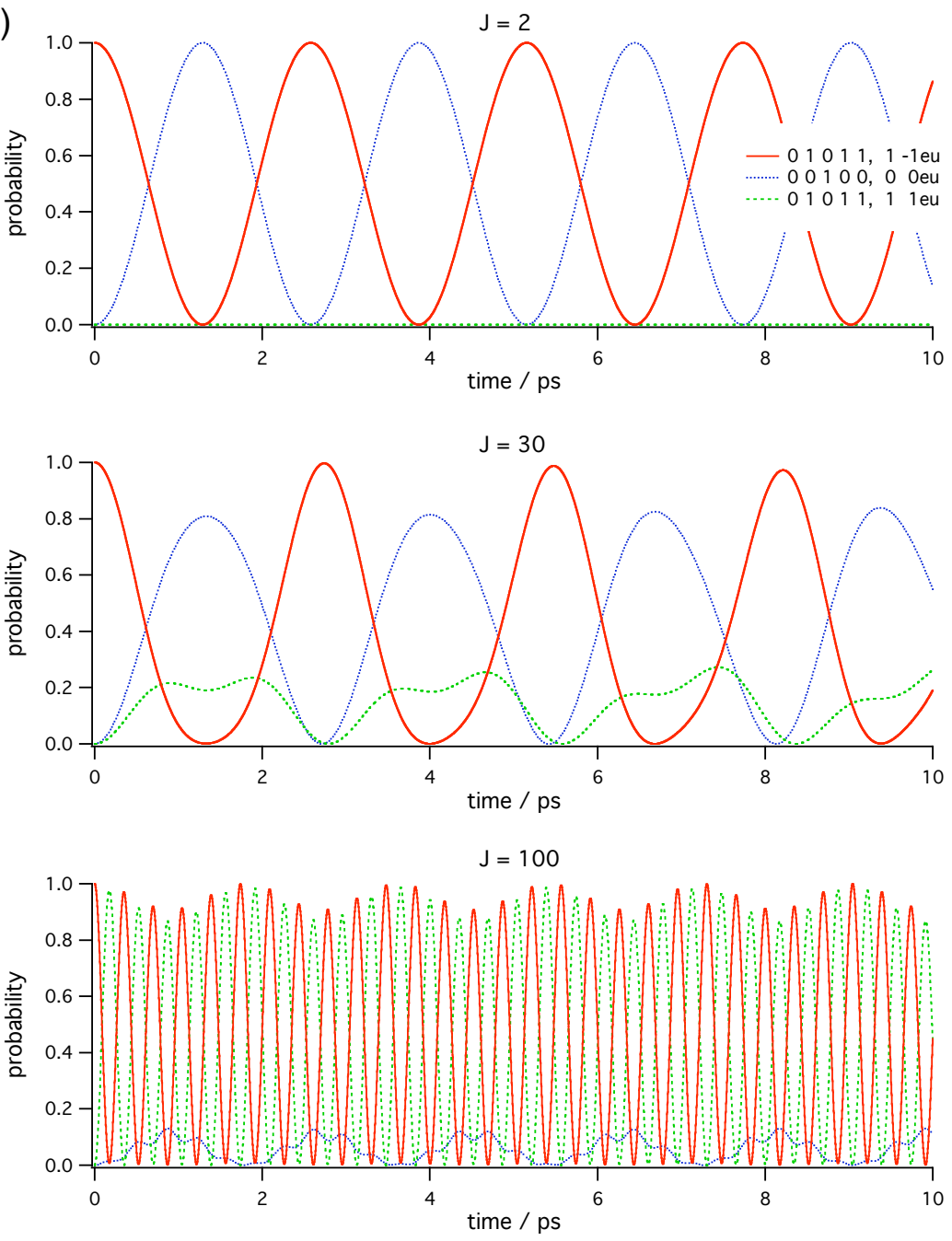

Figure 3: Illustration of the effects of the rotational $l$-type resonance combined with the $3 / 245$ anharmonic resonance in ${ }^{12} \mathrm{C}_{2} \mathrm{H}_{2}, \tilde{X}^{1} \Sigma_{g}^{+}$, selecting the $\left\{1,5, k_{e}, e, u\right\}$ polyad with the bright state taken to be the $(01011,1-1)$, $\Sigma_{e u}$ zeroth-order substate:

(a) Reduced energy graph of the sub-states in the polyad as a function of $J(J+1)$. The eigenstates (black) are labeled I to III according to their energies. The zeroth-order sub-states shown in color and identified on the left as $\left(v_{1} v_{2} v_{3} v_{4} v_{5}, l_{4} l_{5} e u\right)$

(b) Squared coefficients of the bright state in the composition of the eigenstates of the polyad, plotted as a function of $J$. The numbering and line marker styles in parts (a) and (b) are related to the eigenstate label and are internally consistent. The zeroth-order bright sub-state is indicated as $\left(v_{1} v_{2} v_{3} v_{4} v_{5}, l_{4} l_{5} \mathrm{e} / \mathrm{f} \mathrm{g} / \mathrm{u}\right)$;

(c) Calculated time-dependent probability of the basis states for $J=2$ (upper), 30 (medium) and 100 (lower). At $\mathrm{t}=0$, the bright state (red) begins at $P_{l}(t)=1.0$. The color scheme distinguishes the zeroth-order sub-states according to order of their appearance in the time evolution. The color and marker styles for the zeroth-order sub-states are the same in parts (a) and (c). 
$E_{V R}-1.173000 \mathrm{~J}(\mathrm{~J}+1)+1.626110^{-6} \mathrm{~J}^{2}(\mathrm{~J}+1)^{2}\left(\mathrm{~cm}^{-1}\right)$

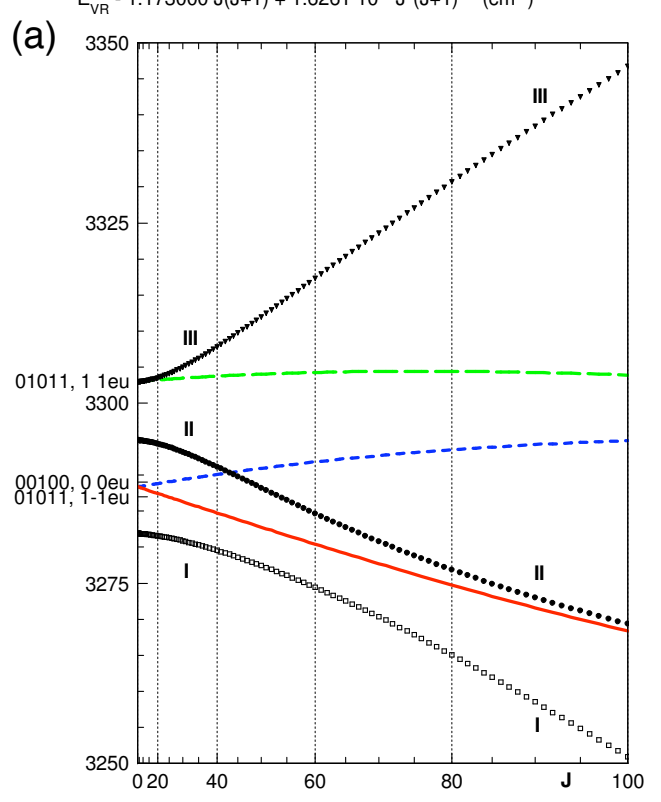

(b)

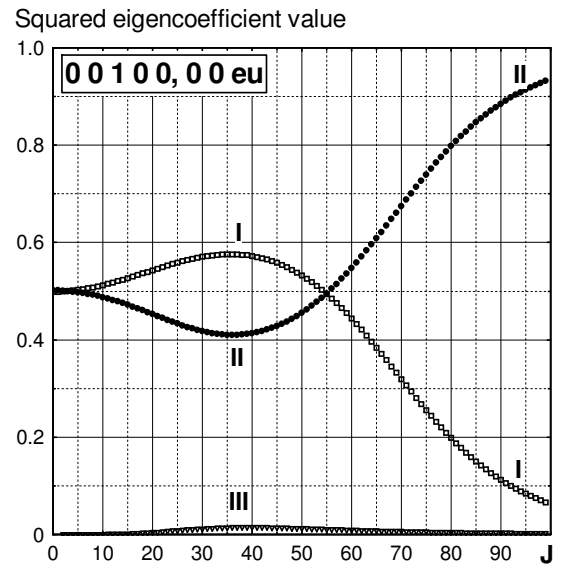

(c)

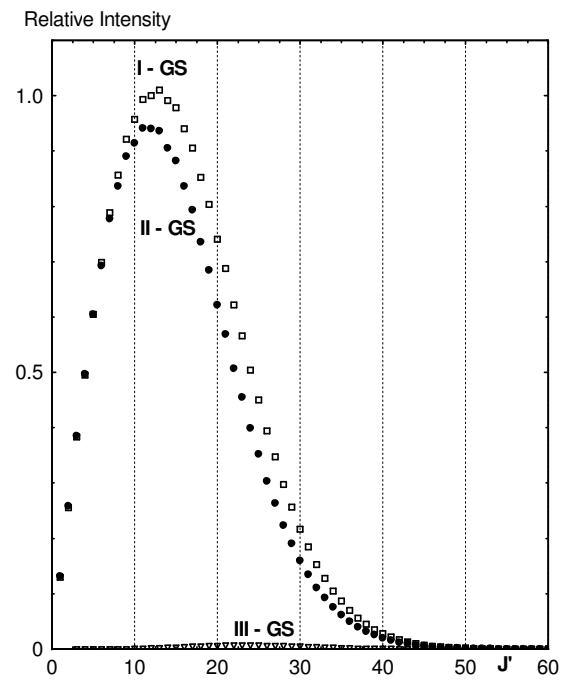

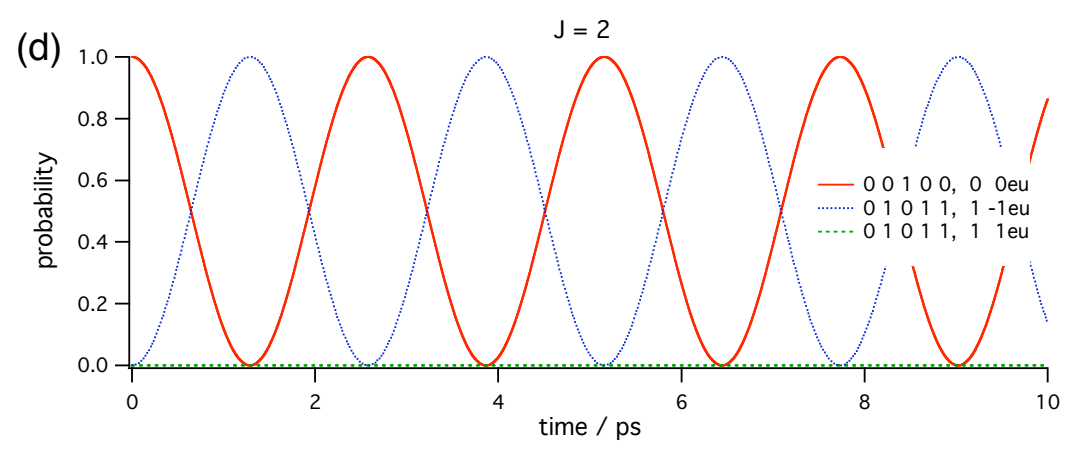
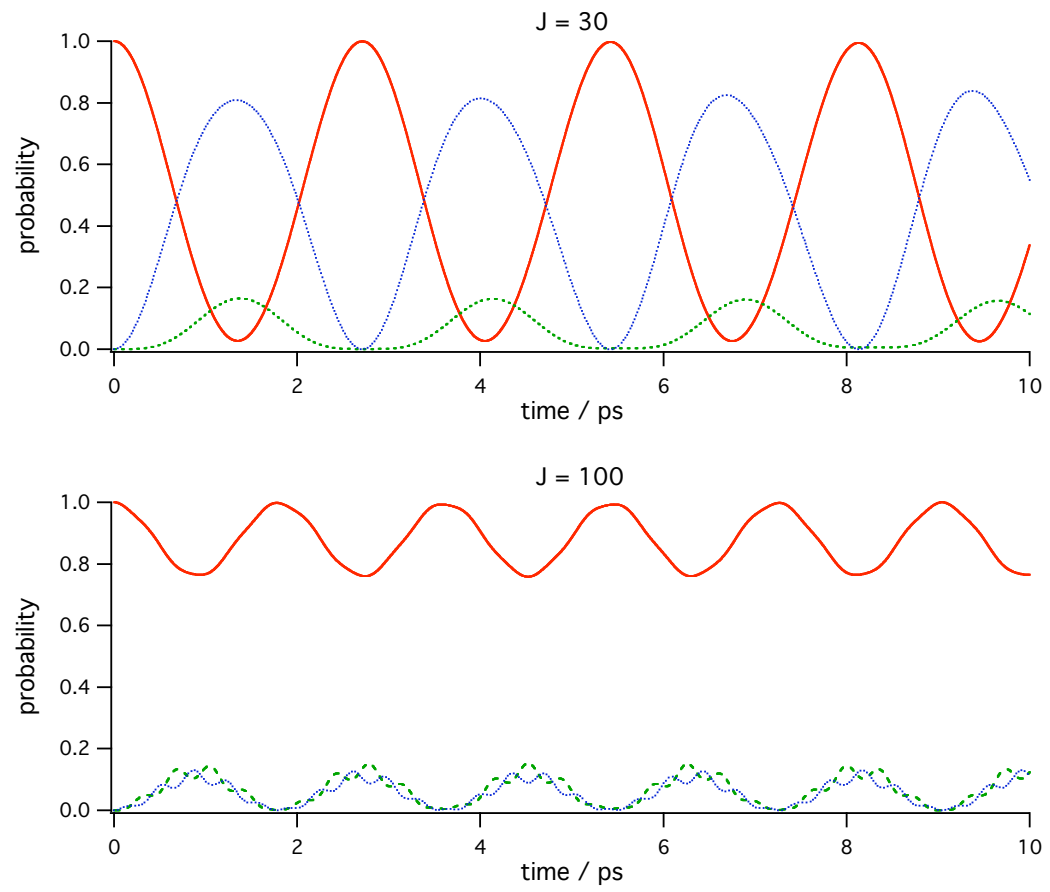

Figure 4: Illustration of the effects of the rotational $l$-type resonance combined to the $3 / 245$ anharmonic resonance in ${ }^{12} \mathrm{C}_{2} \mathrm{H}_{2}, \tilde{X}^{1} \Sigma_{g}^{+}$, selecting the $\left\{1,5, k_{e}, e, u\right\}$ polyad with the bright state taken to be the $(00100,00), \Sigma_{\text {eu }}$ zeroth-order sub-state:

(a) Reduced energy graph of the sub-states in the polyad as a function of $J(J+1)$. The eigenstates (black) are labeled I to III according to the ordering of the energy of the corresponding eigenvalues. The zeroth-order sub-states are colored and identified on the left as $\left(v_{1} v_{2} v_{3} v_{4} v_{5}, l_{4} l_{5} e u\right)$;

(b) Squared coefficients of the bright state in the composition of the eigenstates of the polyad, plotted as a function of $J$. The numbering and line marker styles in parts (a) and (b) are related to the eigenstate label and internally consistent. The zeroth-order bright sub-state is indicated as $\left(v_{1} v_{2} v_{3} v_{4} v_{5}, l_{4} l_{5} \mathrm{e} / \mathrm{f} \mathrm{g} / \mathrm{u}\right)$;

(c) Calculated relative intensities of the $R$-branch lines in the I, II and III $\leftarrow$ GS IR absorption bands. The simulated intensities are given in terms of the upper $J$ quantum number $(J)$ for a temperature of 500 $\mathrm{K}$. The 3:1 intensity alternation due to ortho and para acetylene has been cancelled by multiplying the lower intensity lines by a factor 3 ;

(d) Calculated time-dependent probability of the basis states for $J$ $=2$ (upper), 30 (medium) and 100 (lower). At $\mathrm{t}=0$, the bright state (red) begins at $P_{l}(t)=1.0$. The color scheme distinguishes the zerothorder sub-states according to order of their appearance in the time evolution. The color and marker styles for the zeroth-order sub-states are the same in parts (a) and (d). 
(a) $\mathrm{E}_{\mathrm{VR}}-1.173000 \mathrm{~J}(\mathrm{~J}+1)+1.626110^{-6} \mathrm{~J}^{2}(\mathrm{~J}+1)^{2}\left(\mathrm{~cm}^{-1}\right)$

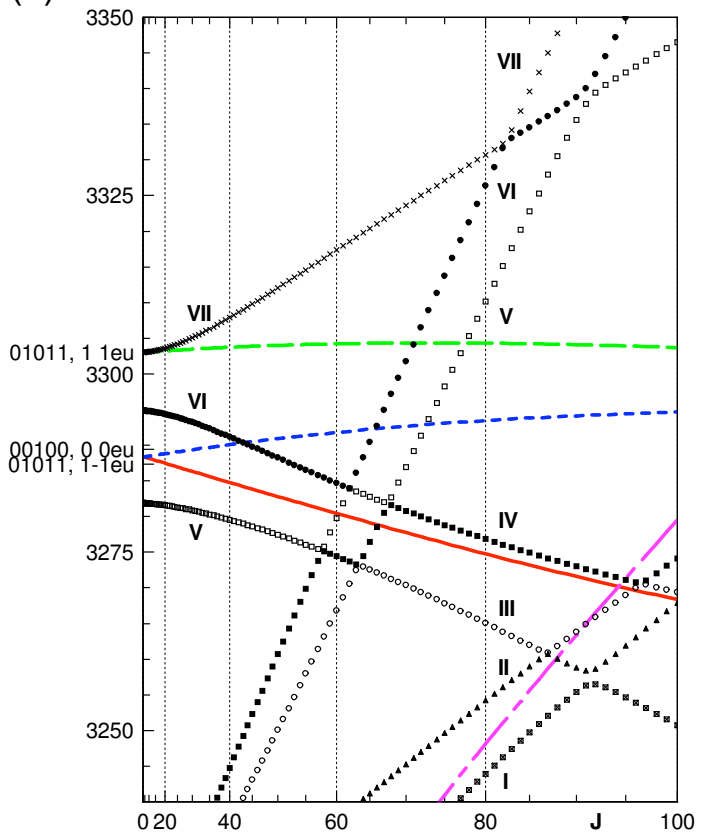

(b)

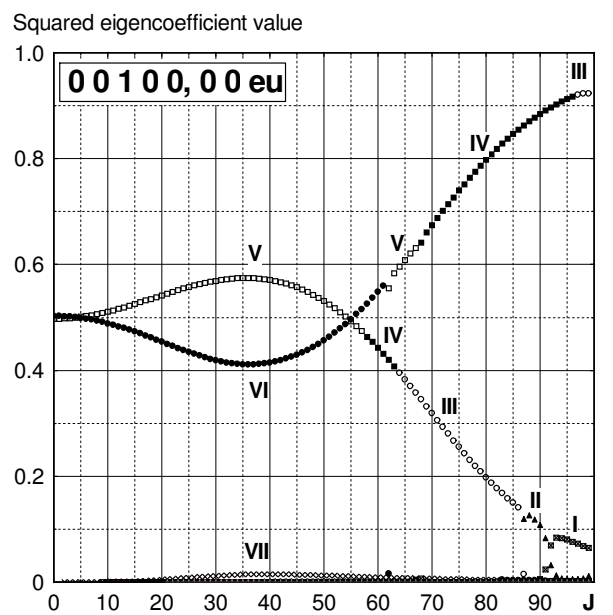

(c)

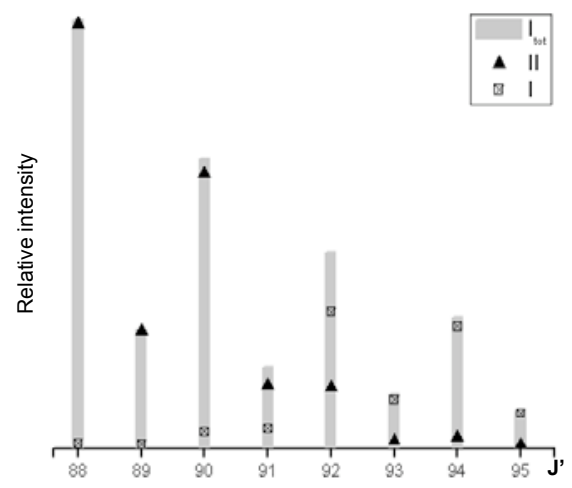

(d)
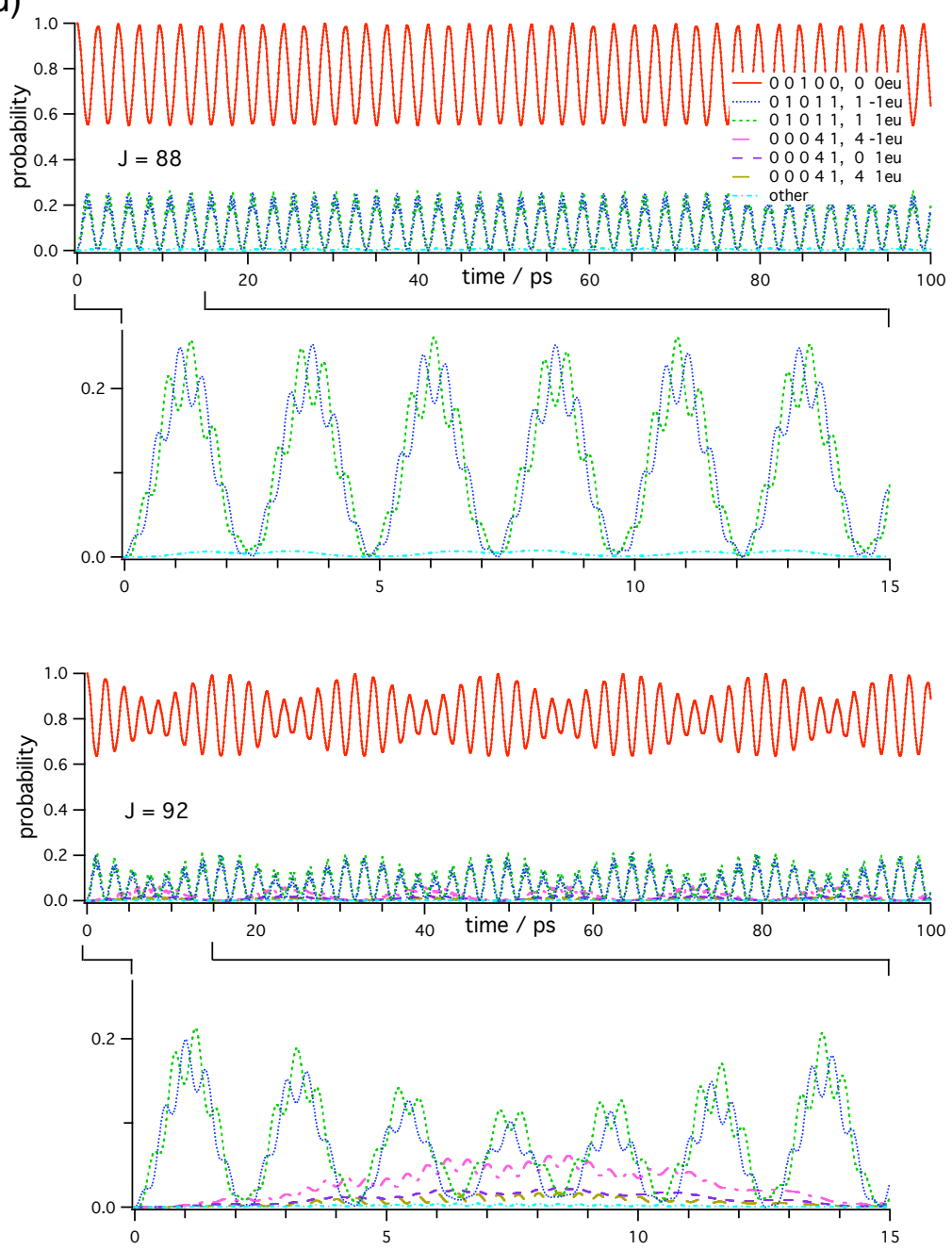

Figure 5: Illustration of the effects of the 2/444 Coriolis resonance combined to rotational $l$-type resonance and 3/245 anharmonic resonance in ${ }^{12} \mathrm{C}_{2} \mathrm{H}_{2}, \tilde{X}^{1} \Sigma_{\sigma}^{+}$, selecting the $\{5, e, u\}$ polyad with the bright state taken to be the $(00100,00), \Sigma_{\text {eu }}$ zeroth-order sub-state:

(a) Reduced energy graph of selected sub-states in the polyad as a function of $J(J+1)$. The eigenstates are identified by the marker style (black) and are labeled I to VII according to their energies. The three most relevant zeroth-order sub-states are colored and identified on the left as $\left(v_{1} v_{2} v_{3} v_{4} v_{5}, l_{4} l_{5} e u\right)$;

(b) Squared coefficients of the eigenvector in the composition of the eigenstates in the polyad, as a function of $J$. The numbering and line marker styles in parts (a) and (b) are related to the eigenstate label and internally consistent. The zeroth-order bright sub-state is indicated as $\left(v_{1} v_{2} v_{3} v_{4} v_{5}, l_{4} l_{5} \mathrm{e} / \mathrm{f} \mathrm{g} / \mathrm{u}\right)$;

(c) Resulting intensity borrowing on related $R$-branch lines simulated at a temperature of $2,000 \mathrm{~K}$, in function of the upper $J$ quantum number $\left(J^{\prime}\right)$. The two major contributions to the intensity are summed up, defining the grey bar;

(d) Calculated time-dependent probability of the basis states for the Coriolis perturbed $J=92$ level (lower), compared $J=88$ level (upper), which is not Coriolis-perturbed. Portions of the graphs shown on an expanded time and probability scales. The color and marker styles for the zeroth-order sub-states are the same in parts (a) and (d). 

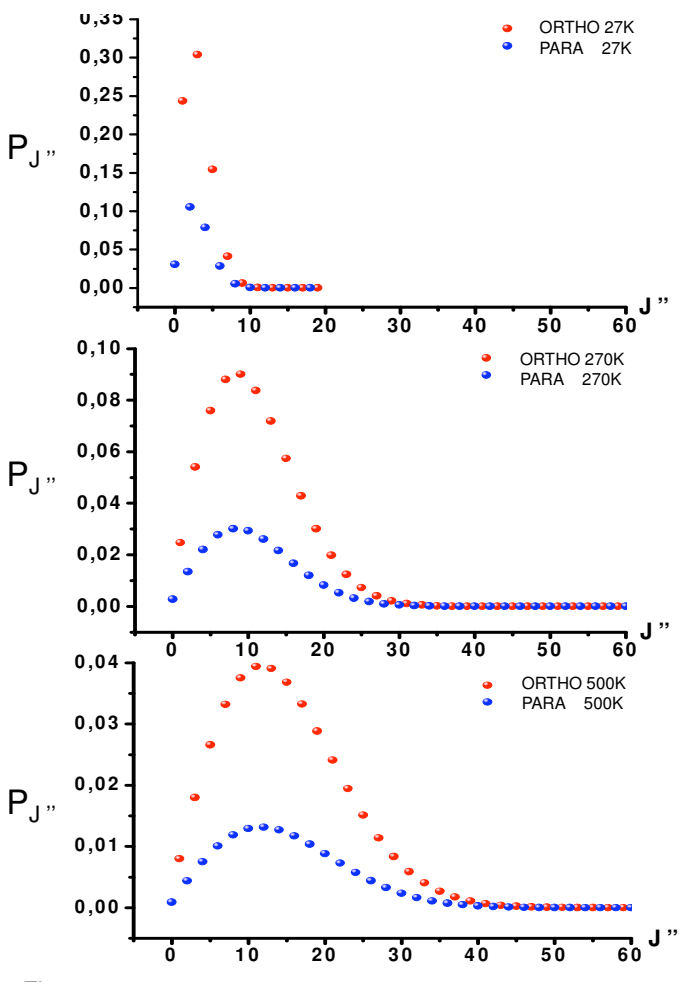

Figure 6: Population distribution in the rotational levels of the GS in ${ }^{12} \mathrm{C}_{2} \mathrm{H}_{2}, \tilde{X}^{1} \Sigma_{g}^{+}$, at 27,270 and $500 \mathrm{~K}$.

The ortho and para distributions are color coded. 
(a) $\mathrm{E}_{\mathrm{VR}}-1.175076 \mathrm{~J}(\mathrm{~J}+1)+1.626110^{-6} \mathrm{~J}^{2}(\mathrm{~J}+1)^{2}\left(\mathrm{~cm}^{-1}\right)$

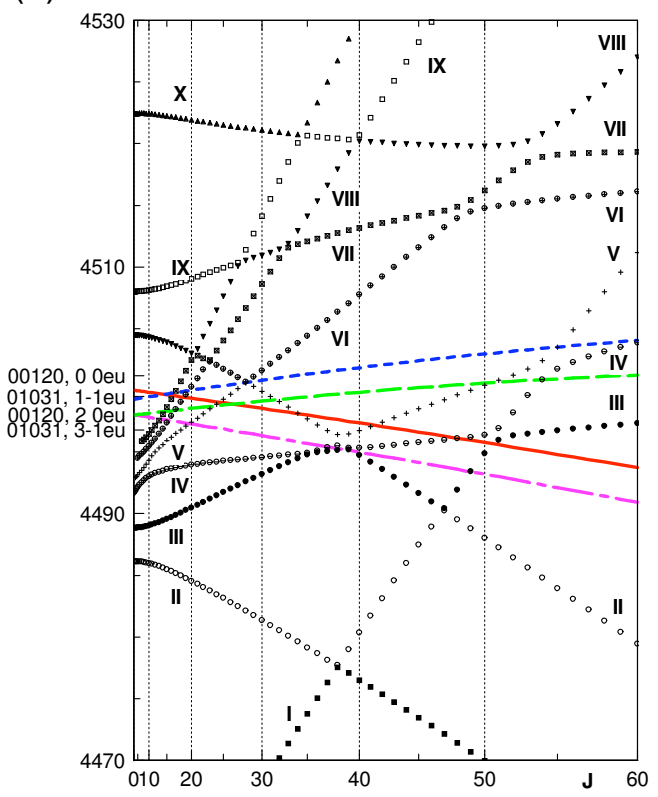

(b) Squared eigencoefficient value

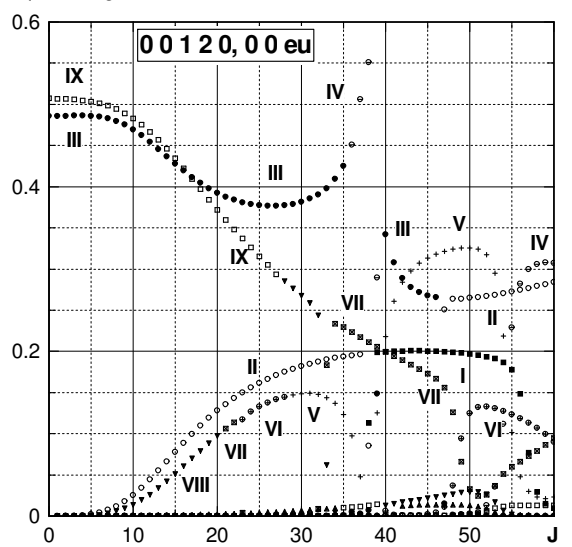

(c)
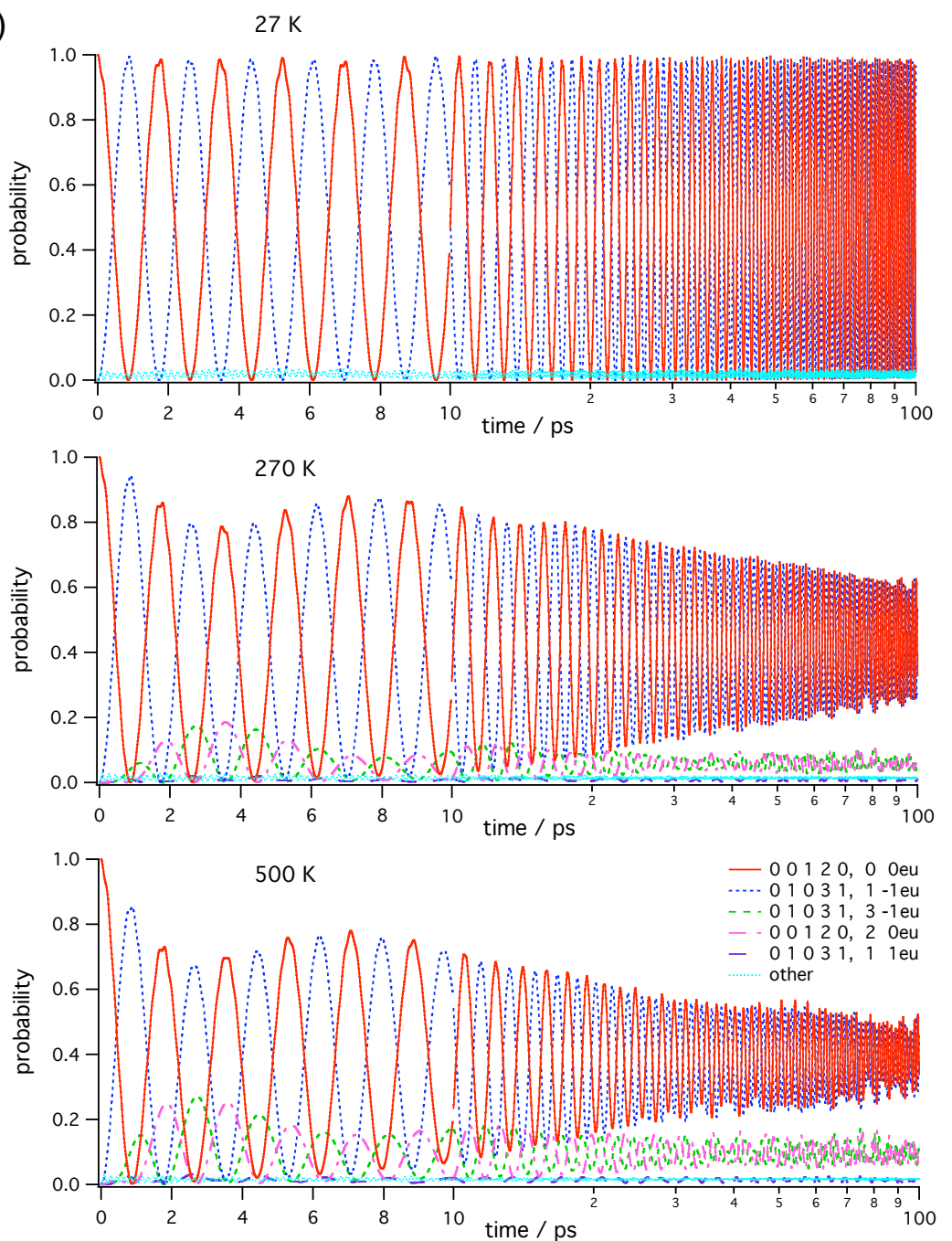

Figure 7: The $\{7, e, u\}$ polyad in ${ }^{12} \mathrm{C}_{2} \mathrm{H}_{2}, \tilde{X}^{1} \Sigma_{g}^{+}$, with the zeroth-order bright sub-state taken to be $(00120,00), \Sigma_{\text {eu: }}$ :

(a) Reduced energy graph of selected sub-states as function of $J(J+1)$. The eigenstates are identified by the marker style (black) and are labeled I to X according to their energies. The four most relevant zeroth-order sub-states are colored and identified on the left as $\left(v_{1} v_{2} v_{3} v_{4} v_{5}, l_{4} l_{5} e u\right)$;

(b) Squared coefficients of the zeroth-order bright sub-state eigenvector in the composition of the eigenstates in the polyad, as a function of $J$. The numbering and line marker styles in parts (a) and (b) are related to the eigenstate label and are internally consistent. The zeroth-order bright sub-state is indicated as $\left(v_{1} v_{2} v_{3} v_{4} v_{5}, l_{4} l_{5} \mathrm{e} / \mathrm{f} \mathrm{g} / \mathrm{u}\right)$;

(c) Calculated time-dependent probability of the basis states for a thermal averaged population in the upper zerothorder bright sub-state, for $\mathrm{T}=27$ (upper), 270 (middle) and 500 (lower) K. The color code and line marker styles distinguish zeroth-order sub-states according to their successive rank in the time evolution. The color and marker styles for the zeroth-order sub-states are identical in parts (a) and (c). 
(a) $\mathrm{E}_{\mathrm{VR}}-1.180155 \mathrm{~J}(\mathrm{~J}+1)+1.626110^{-6} \mathrm{~J}^{2}(\mathrm{~J}+1)^{2} \quad\left(\mathrm{~cm}^{-1}\right)$

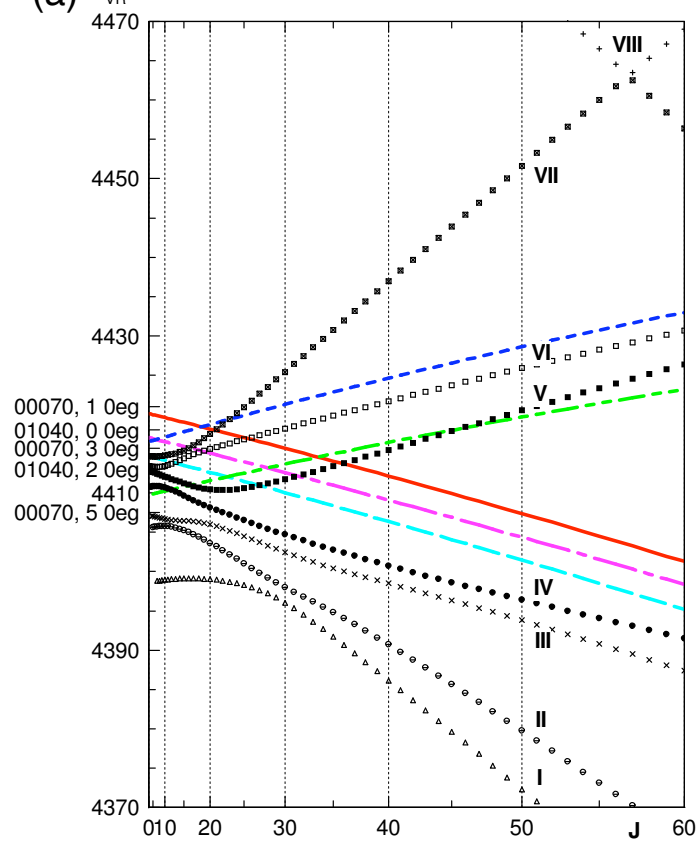

(b) Squared eigencoefficient value

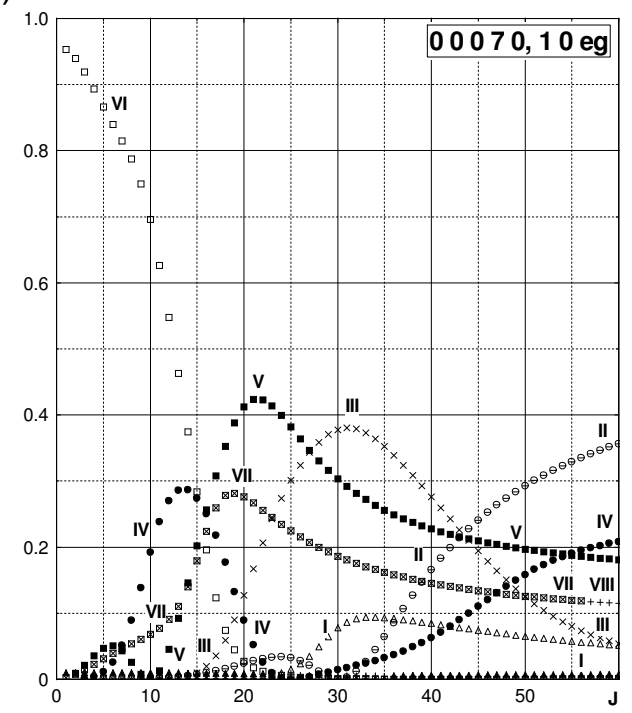

(c)
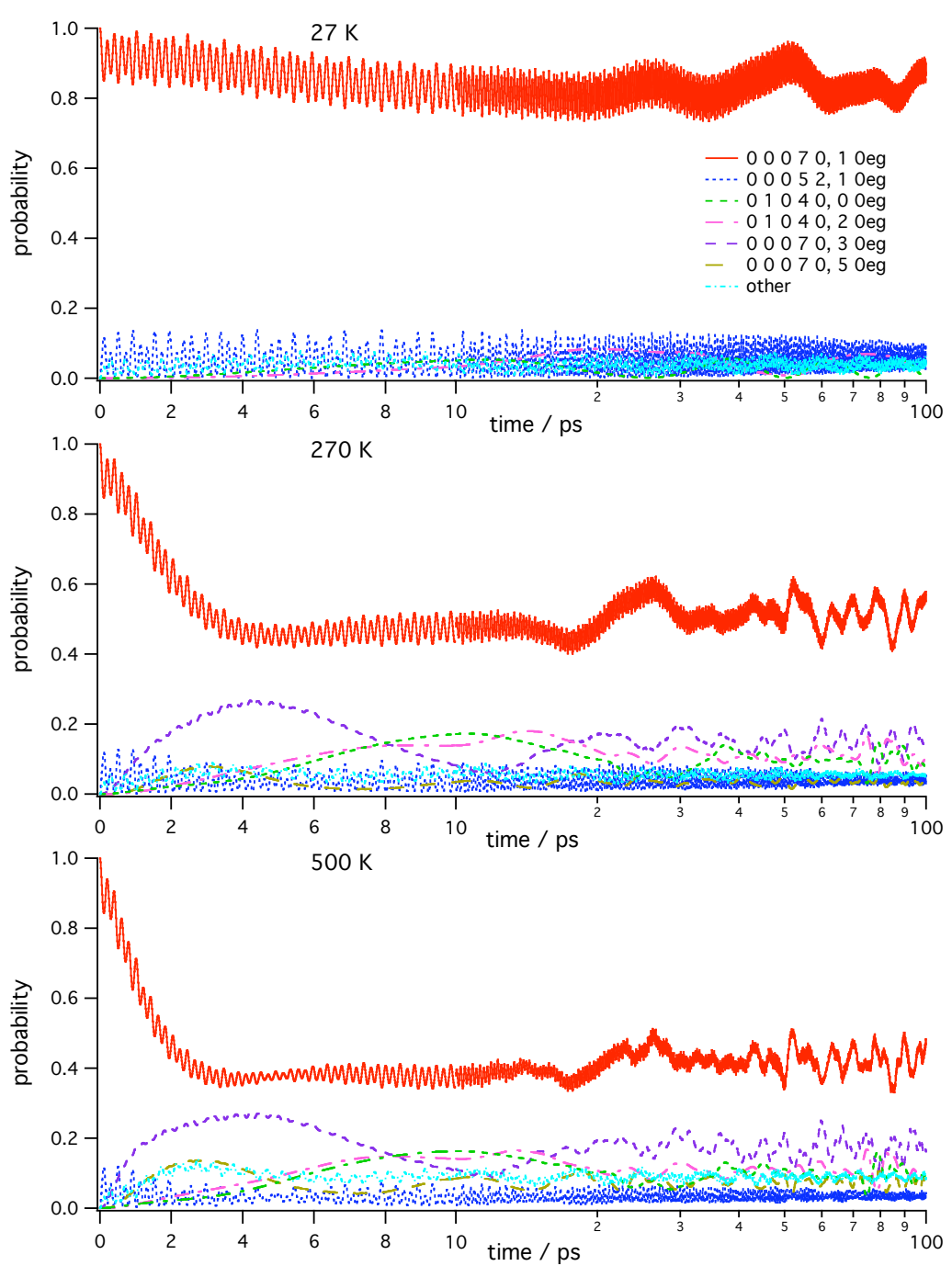

Figure 8: The $\{7, e, g\}$ polyad in ${ }^{12} \mathrm{C}_{2} \mathrm{H}_{2}, \tilde{X}^{1} \Sigma_{g}^{+}$, with the zeroth-order bright sub-state taken to be $(00070,10), \Pi_{e g}$ :

(a) Reduced energy graph of selected sub-states in function of $J(J+1)$. The eigenstates are identified by the marker style (black) and are labeled I to VIII according to their energies. The five most relevant zeroth-order sub-states are colored and identified on the left as $\left(v_{1} v_{2} v_{3} v_{4} v_{5}, l_{4} l_{5} e u\right)$;

(b) Squared coefficients of the $(00070,10), \Pi_{e g}$, zeroth-order sub-state eigenvector in the composition of the other eigenstates in the polyad, as a function of $J$. The numbering and line marker styles in parts (a) and (b) are related to the eigenstate label and are internally consistent. The zeroth-order bright sub-state is indicated as $\left(v_{1} v_{2} v_{3} v_{4} v_{5}, l_{4} l_{5} \mathrm{e} / \mathrm{f} \mathrm{g} / \mathrm{u}\right)$;

(c) Calculated time-dependent probability of the basis states for a thermal averaged population in the upper zerothorder bright sub-state, for $\mathrm{T}=27$ (upper), 270 (middle) and 500 (lower) K. The color scheme and line marker styles distinguish zeroth-order sub-states according to their order of appearance in the time evolution. The color and marker styles for the zeroth-order sub-states are the same in parts (a) and (c). 